\title{
Dispersive Computational Continua
}

\author{
Vasilina Filonova ${ }^{\mathrm{a}}$, Dimitrios Fafalis ${ }^{\mathrm{a}}$, Jacob Fish ${ }^{\mathrm{a}, *}$ \\ ${ }^{a}$ Columbia University, New York, USA
}

\begin{abstract}
The two primary objectives of the present manuscript are: (i) to develop a variant of the computational continua formulation $\left(\mathrm{C}^{2}\right)$ with outstanding dispersive properties, and (ii) to conduct a rigorous dispersion analysis of it. The ability of the $\mathrm{C}^{2}$ formulation to capture dispersive behavior stems from its underlying formulation, which does not explicitly assume scale separation and accounts for microstructures of finite size. The dispersion study in heterogeneous elastic media with periodic microstructure has been conducted using both analytical and numerical approaches. The so-called analytical dispersion analysis is based on the Floquet-Bloch wave solution, while the numerical dispersion analysis is based on the modal analysis of the discrete coupled fine-coarse-scale equations. The dispersive curves obtained from the dispersive $\mathrm{C}^{2}$ formulations were compared with the classical exact Floquet-Bloch wave solution, hereafter referred to as the reference dispersive curve. It has been observed that in the case of the unit cell sizes being either half of the coarse-scale element size or equal to it, the dispersive curves obtained by the dispersive $\mathrm{C}^{2}$ formulation are practically identical to the reference solution. For other cases, the dispersive $\mathrm{C}^{2}$ solution is in good agreement with the reference solution provided that the wavelength is resolved by at least two coarse-scale quadratic elements. The dispersion analysis results have been further verified by the wave propagation problem in a periodic heterogeneous medium with a wavelength comparable to the microstructural size.
\end{abstract}

Keywords: Homogenization, Computational continua, Dispersion analysis, High-frequency, FloquetBloch wave

\section{Introduction}

Wave propagation in periodic structures such as layered media or crystals may exhibit remarkable properties. For instance, when the microstructural size is comparable to the wavelength, the wave reflection and refraction from micro-constituent interfaces, known as the dispersion effect, may become significant enough to influence the overall dynamic response. In recent years, consideration of the wave phenomena in composites has motivated development of new materials, such as metamaterials [1] and photonic crystals [2].

Developing computational approaches aimed at resolving strong dispersion effects in heterogeneous media where the microstructural size is comparable to the length of the traveling signal remains of interest to the scientific community almost half a century after the pioneering

*Corresponding author. Address: Columbia University, Department of Civil Engineering and Engineering Mechanics, 610 Seeley W. Mudd, 500 West $120^{\text {th }}$ Street, New York, NY 10027, USA, Tel.: +1 212854 3143; fax:

+1 2128546267 .

Email addresses: fishj@columbia.edu (J. Fish),vf2197@columbia.edu (V. Filonova),df2472@columbia.edu (D. Fafalis). 
work of Achenbach and his associates [3,4]. This high-frequency case goes beyond the long wavelength that is typically considered in first order homogenization methods, or so-called $\mathrm{O}(1)$ homogenization. It is well-known that $\mathrm{O}(1)$ homogenization (and equivalently effective medium theories) cannot predict the reflection and refraction of stress waves that give rise to the dispersion and attenuation of waves within a material microstructure $[5,6]$. Consequently, for transient problems the $\mathrm{O}(1)$ homogenization theory and effective medium theories are limited to low frequencies or long wavelengths.

Higher order homogenization methods and equivalently generalized continua theories permit the consideration of higher frequencies within a long wave approximation (see, e.g., $[7,8,9,10,11,12,13,14])$. However, most of the higher order homogenization theories introduce secular terms that grow unbounded in time $[9,15,16]$. The secularity of the asymptotic expansion can be eliminated by introducing slow time scale(s) aimed at capturing the long-time effects of dispersion in addition to the fast spatial scale aimed at the spatial resolution of the microstructure (see $[9,15,16])$. The high-frequency homogenization method introduced in $[17,18]$ goes beyond the long-wavelength approximation in modeling transient interactions in heterogeneous media. It resolves the high frequencies of strongly oscillating fields and consequently is capable of giving the Bloch mode spectrum near the edge of the Brillouin zone.

Dispersive continua formulations have also been successfully derived without resorting to higher order theories, with their well-known shortcomings, such as higher order continuity and often non-physical boundary conditions. Noteworthy are the averaging techniques based on various self-consistent schemes that provide dynamic effective constitutive relations of elastic composites [7,19,20,21,22], methods based on equivalent quasi-dynamic unit cell problems $[23,24,25]$, and approaches aimed at extending the Hill-Mandel macro-homogeneity condition to high frequency dynamics [26] along the lines of the Virial formula [27] used in molecular dynamics.

To quantify the dispersive properties of a specific method, it is common in practice to compute the dispersive relation for a one-dimensional harmonic wave and compare the result with a known exact solution. The wave equation for the infinite layered periodic structure with piecewise elastic material properties can be analytically described by a Floquet (or Bloch)-type wave $[28,29]$. A closed-form solution was originally reported in [30,31,32] and later utilized by other investigators (e.g., $[12,33,34])$. The natural frequencies (modal) analysis is commonly used to study the dispersion properties of numerical (discretized) solutions.

The primary objectives of the present study are to extend the computational continua $\left(\mathrm{C}^{2}\right)$ formulation [35,36,37] to a high-frequency transient regime and analyze its dispersive properties. The $\mathrm{C}^{2}$ formulation has in principle the potential to capture dispersive behavior, since, unlike various homogenization theories, it does not explicitly assume scale separation and at the same time considers unit cells of a finite size. Consequently, the method is potentially wellsuited for high-frequency wave propagation problems in heterogeneous media where microstructural size is comparable to the wavelength of a traveling signal.

Most of the dispersive continuum theories for composite materials are limited to linear elastic materials (see $[7,8,9,11,12,13,14,18,19,25])$. Studies of nonlinear dispersive formulations are rare (see $[23,25,38,39,40,41])$. Although the focus of the present manuscript is on dispersion analysis of linear elastic materials, the $\mathrm{C}^{2}$ approach has been extensively studied for nonlinear steady state long-wavelength problems [35-37].

The structure of the present manuscript is as follows. Section 2 briefly outlines the computational continua formulation for transient problems in multiple dimensions. In Section 3, 
the dispersion relation for the dispersive $C^{2}$ formulation is derived in closed form by utilizing the Floquet-Bloch type solution. In Sections 4.2, the natural frequency (modal) analysis of coupled discrete fine-coarse-scale equations is conducted to verify the analytical dispersion study presented in Section 3. The stop and pass frequency bands are shown in Section 4.3. Finally, a numerical example of a high-frequency wave of a length comparable to the microstructural size propagating in a periodic elastic rod is presented in Section 4.3. Summary and future research directions conclude the manuscript in Section 5.

\section{Formulation of the dispersive computational continua}

Consider a wave propagation problem in a periodic composite domain $\Omega^{\zeta}$

$$
\begin{aligned}
& \nabla \cdot \boldsymbol{\sigma}^{\zeta}(\mathbf{x}, t) \boldsymbol{x}+\mathbf{u}^{\zeta}(\mathbf{x}, t)=\rho^{\zeta}()^{\cdot \zeta}(, t), \\
& \boldsymbol{\sigma}^{\zeta}(\mathbf{x}, t)=\boldsymbol{F}\left(\boldsymbol{\varepsilon}^{\zeta}(\mathbf{x}, t)\right), \\
& \boldsymbol{\varepsilon}^{\zeta}(\mathbf{x}, t)=\frac{1}{2}\left(\nabla \mathbf{u}^{\zeta}+\nabla \mathbf{u}^{\zeta T}\right),
\end{aligned}
$$

where (1)a denotes the balance of linear momentum; (1)b the stress-strain constitutive relation defined by $\boldsymbol{F}$, which in general may depend on state variables and deformation history; and (1)c the small deformation strain-displacement relation, which, for simplicity, is considered hereafter. $\nabla$ is the gradient operator; the superscript $T$ denotes the transpose, and the superimposed dot denotes the material time derivative. $\mathbf{x}$ is a global spatial coordinate defined in $\Omega^{\zeta}$ and $t$ is the time; $\boldsymbol{\sigma}^{\zeta}$ is the overall Cauchy stress; $\mathbf{u}^{\zeta}$ the overall displacement; $\boldsymbol{\varepsilon}^{\zeta}$ the overall small strain; and $\mathbf{b}^{\zeta}$ the overall body force. The superscript $\zeta$ denotes the existence of the microstructure; $\chi=\mathbf{x}-\hat{\mathbf{x}}_{I}$ is a local coordinate defined over the unit cell domain (or representative volume element), $\Theta_{I}$, with $\hat{\mathbf{x}}_{I}$ being the centroid of the $I$-th unit cell. Thus, various fields can be described as a function of the unit cell centroid and the local coordinate, i.e., $f^{\zeta}(\mathbf{x}, t)=f^{\zeta}\left(\hat{\mathbf{x}}_{I}, t\right)$, where $f^{\zeta}$ denotes an arbitrary function. Material properties, such as elastic stiffness tensor $\mathbf{L}^{\zeta}$ (relating $\sigma^{\zeta}=\mathbf{L}^{\zeta} \boldsymbol{\varepsilon}^{\zeta}$ for linear elasticity problems) and density $\rho^{\zeta}$, depend in general on the position of the unit cell centroid and the local coordinate. For simplicity, in the following we assume that all unit cells are identical and thus $\mathbf{L}^{\zeta}()$ and $\rho^{\zeta}(\chi)$.

Following the variational multiscale method [42], the $C^{2}$ formulation $[35,36]$ employs an additive decomposition of overall fields by which the overall displacement $\mathbf{u}^{\zeta} \chi \hat{\mathbf{x}}_{I}$,,$\left.t\right)$ is decomposed into the coarse-scale displacement, $\mathbf{u}^{c}\left(x \hat{\mathbf{x}}_{I}, t\right)$, and the fine-scale perturbation, $\mathbf{u}^{*}\left(\hat{\mathbf{x}}_{I},, t\right)$. For the second order $\mathrm{C}^{2}$ formulation considered hereafter, the coarse-scale displacement $\mathbf{u}^{c}$ is approximated by a quadratic function over the unit cell domain $\Theta_{I}$, giving rise to a linear variation of the coarse-scale strain 


$$
\begin{aligned}
& \mathbf{u}^{\zeta}=\mathbf{u}^{c}+\mathbf{u}^{*} ; \quad \boldsymbol{\varepsilon}^{c} \equiv \frac{1}{2}\left(\nabla \mathbf{u}^{c}+\nabla \mathbf{u}^{c T}\right) ; \quad \boldsymbol{\varepsilon}^{*} \equiv \frac{1}{2}\left(\nabla \mathbf{u}^{*}+\nabla \mathbf{u}^{* T}\right), \\
& \boldsymbol{\varepsilon}^{\zeta}=\boldsymbol{\varepsilon}^{c}+\boldsymbol{\varepsilon}^{*} ; \boldsymbol{\varepsilon}^{c} \square \overline{\boldsymbol{\varepsilon}}+\bar{\nabla} \overline{\boldsymbol{\varepsilon}} \boldsymbol{\chi}, \\
& \overline{\boldsymbol{\varepsilon}}\left(\hat{\mathbf{x}}_{I} \boldsymbol{\not} \boldsymbol{t}\right) \equiv\left\langle\boldsymbol{\varepsilon}^{c}\left(\hat{\mathbf{x}}_{I},, \mathbf{\mathbf { x }}\right)\right\rangle_{\Theta_{I}} ; \quad \bar{\nabla} \overline{\boldsymbol{\varepsilon}}\left({ }^{\wedge} \mathbf{x}, t\right) \equiv\left\langle\nabla \boldsymbol{\varepsilon}^{c}\left({ }_{I},, t\right)\right\rangle_{\Theta_{I}},
\end{aligned}
$$

where $\varepsilon^{c}$ and $\varepsilon^{*}$ are the coarse-scale and perturbation strains, respectively ; $\bar{\varepsilon}$ and $\bar{\nabla} \bar{\varepsilon}$ are the average coarse-scale strain and its gradient over the unit cell domain. The averaging operator $\langle f\rangle_{V}$ over an arbitrary domain $V$ is defined as

$$
\langle f\rangle_{V}=\frac{1}{|V|} \int_{V} f d V
$$

Multiplying (1)a by an arbitrary $C^{0}$ continuous test function $\mathbf{w}(\mathbf{x}, t) \in V$ that satisfies homogeneous boundary conditions on essential boundary $\partial \Omega^{u \zeta}$, subsequently integrating over the composite domain $\Omega^{\zeta}$ and finally integrating by parts, yields the classical single-scale weak form

$$
\int_{\Omega^{\zeta}}(\nabla \mathbf{w})^{T} \boldsymbol{\sigma}^{\zeta} d \Omega+\int_{\Omega^{\zeta}} \rho^{\zeta} \mathbf{w}^{T} \ddot{\mathbf{u}}^{\zeta} d \Omega=\int_{\partial \Omega^{t_{\zeta}}} \mathbf{w}^{T} \mathbf{t}^{\zeta} d \Gamma+\int_{\Omega^{\zeta}} \mathbf{w}^{T} \mathbf{b}^{\zeta} d \Omega \quad \forall \mathbf{w} \in V,
$$

where $\mathbf{t}^{\zeta}=\boldsymbol{\sigma}^{\zeta} \mathbf{n}^{\zeta}$ is an overall traction defined on the natural boundary $\partial \Omega^{t \zeta}$, such that $\partial \Omega^{t \zeta} \cup \partial \Omega^{u \zeta}=\partial \Omega^{\zeta}$ and $\partial \Omega^{t \zeta} \bigcap \partial \Omega^{u \zeta}=0$.

In the next subsections, we describe the weak form of the governing equation for the coarseand fine-scale problems and elaborate on the test function space $V$.

\subsection{Nonlocal quadrature and the coarse-scale problem}

One of the salient features of the $\mathrm{C}^{2}$ formulation is nonlocal quadrature [36,35,37], which is a variant of the Gauss quadrature aimed at accounting for unit cells of a finite size comparable with a characteristic coarse-scale element size. By this approach, integrals over a composite domain $\Omega^{\zeta}$ in (3) are approximated by integration over the so-called computational continua domain $\Omega^{c}$, defined as a disjoint union of $\hat{N}$ computational unit cells $\Omega^{c}=\coprod_{I=1}^{\hat{N}} \Theta_{I}$. The nonlocal quadrature over a single coarse-scale finite element domain $\Omega^{e, \zeta}$ is defined as

$$
\int_{\Omega^{e, \zeta}} f^{\zeta}(\mathbf{x}) \not d \Omega^{e}=\sum_{I=1}^{n^{i p}} v_{I} J_{I}\left\langle f^{\zeta}\left(\hat{\mathbf{x}}_{I},\right)\right\rangle_{\Theta_{I}},
$$

where $v_{I}$ is the nonlocal quadrature weight; $J_{I}$ the $I$-th unit cell Jacobian mapping the coarsescale element into a bi-unit parent element domain; $\hat{\mathbf{x}}_{I}$ the coordinate of the nonlocal quadrature point centroid; and $n^{i p}$ the number of nonlocal quadrature points in a single coarse-scale finite element.

Applying the nonlocal quadrature (4) to the weak form (3) discretized by $n^{e l}$ coarse-scale elements yields 


$$
\begin{aligned}
& \sum_{a=1}^{n^{e l}} \sum_{I=1}^{n^{i p}} v_{a I} J_{a I}\left\langle\left(\nabla \mathbf{w}^{c}\right)^{T} \boldsymbol{\sigma}^{\zeta}+\rho^{\zeta} \mathbf{w}^{c T} \ddot{\mathbf{u}}^{\zeta}\right\rangle_{\Theta_{a I}}=\int_{\partial \Omega^{t \zeta}} \mathbf{w}^{c T} \mathbf{t}^{\zeta} d \Gamma+\sum_{a=1}^{n^{e l}} \sum_{I=1}^{n^{i p}} v_{a I} J_{a I}\left\langle\mathbf{w}^{c T} \mathbf{b}^{\zeta}\right\rangle_{\Theta_{a l}}, \\
& \forall \mathbf{w}^{c} \in V^{c}=\left\{\mathbf{w}^{c} \in C^{0}\left(\Omega^{c}\right), \mathbf{w}^{c}=\mathbf{0} \text { on } \partial \Omega^{u \zeta}\right\},
\end{aligned}
$$

where the total number of nonlocal quadrature points is given by $\hat{N}=n^{e l} n^{i p}$. Note that equations (3) and (5) can accommodate nonlinear kinematics and nonlinear constitutive equations. However, for simplicity, linear elasticity and small deformations are considered herein.

The coarse-scale test function $\mathbf{w}^{c}$ is assumed to be $C^{0}$ continuous on $\Omega^{c}$ and to satisfy homogenous boundary conditions on the essential boundary $\partial \Omega^{u \zeta}$. In equation (5), the subscript aI denotes the $I$-th nonlocal integration point in the $a$-th element.

Hereafter, we consider quadratic one-dimensional coarse-scale elements. The inertia term $\rho^{\zeta} \mathbf{w}^{c T} \ddot{\mathbf{u}}^{\zeta}$ in equation (5) is a fourth order, which requires three quadrature points for exact integration. In some cases, as will be discussed later, we will employ reduced integration. The weights $v_{a I}$ and locations $\hat{\eta}_{a I}$ of either two or three quadrature points in the parent element domain are given by [37,36,35]

$$
\begin{aligned}
& \text { a) } n^{i p}=2: \quad v_{a I}=1 ; \quad \hat{\eta}_{a 1,2}=\mp \sqrt{\frac{1-\left(\zeta_{a I}\right)^{2}}{3}} ; \quad \zeta_{a I} \equiv \frac{\left|\Theta_{a I}\right|}{2 J_{a I}}, \\
& \text { b) } n^{i p}=3: \quad v_{a 1,3}=\frac{5\left[1-\left(\zeta_{a I}\right)^{2}\right]}{3\left[3-7\left(\zeta_{a I}\right)^{2}\right]} ; \quad v_{a 2}=2-2 v_{a 1} ; \quad \hat{\eta}_{a 1,3}=\mp \sqrt{\frac{3-7\left(\zeta_{a I}\right)^{2}}{5}} ; \quad \hat{\eta}_{a 2}=0 .
\end{aligned}
$$

For simplicity, hereafter we consider all unit cells to be of the same size, denoted by $\left|\Theta_{a I}\right| \equiv l$, and all coarse-scale elements to be of the same size as well, denoted by $L^{e l}$. Thus, the ratio of the unit cell size to the coarse-scale element size denoted by $\zeta_{a I}=\zeta \equiv l / L^{e l}$ is fixed. It can be shown from (6) that the so-called size ratio $\zeta$ is bounded by $0 \leq \zeta \leq 1$ for a two-point quadrature and by $0 \leq \zeta \leq 0.5$ for a three-point quadrature.

Fig. 1 depicts a quadratic coarse-scale element with two nonlocal integration points and various unit cell sizes mapped into a parent element domain with a coordinate $\eta$; the Jacobean that maps the physical domain into the parametric domain is constant $J_{a I}=J \equiv L^{e l} / 2$; and the local coordinate in the parent element domain is $r=\eta-\hat{\eta}_{a I}=\chi / J_{a I}$.

Fig. 1. is here

Remark 1. In the computational continua domain $\Omega^{c}$, the computational unit cells are defined by their centroid location, $\hat{\mathbf{x}}_{a I}$, and their size ratio $\zeta$. Note that in general, computational unit cells can overlap or have gaps as shown in Fig. 1a. For instance, for quadratic coarse-scale elements with two integration points (6)a, the unit cells partially overlap for $0.5<\zeta<1$ and fully overlap for $\zeta=1$; there is a gap between unit cells for $0 \leq \zeta<0.5$. When the computational unit cells do not overlap, the disjoint union coincides with the regular union of disjoint sets $\Omega^{c}=\bigcup_{m=1}^{\hat{N}} \hat{\Theta}_{m}, \hat{\Theta}_{m} \cap \hat{\Theta}_{n}=0, \forall m \neq n$. Of particular interest is size ratio $\zeta=0.5$, corresponding to 
$\hat{\eta}_{a 1,2}=\mp 0.5$, in which case the union of disjoint computational unit cells coincides exactly with the physical composite domain $\Omega^{c}=\Omega^{\zeta}$. Another case of interest is $\zeta=1$ where the two computational unit cells coincide at $\hat{\eta}_{a 1,2}=0$. Then, $\zeta=0.5$ and $\zeta=1$ are the only two cases for two-point integration, where the $\mathrm{C}^{2}$ solution can be compared with a single-scale solution in terms of the physical position of the unit cells. Similarly, we find that for three-point integration (6)b, the size ratio $\zeta=1 / 3$ provides the quadrature point locations $\hat{\eta}_{a 1,3}=\mp 3 / 2, \hat{\eta}_{a 2}=0$ which are consistent with the physical positions of unit cells, i.e. $\Omega^{c}=\Omega^{\zeta}$.

Remark 2. The necessary number of integration points for the nonlocal and Gauss quadrature schemes are identical, i.e., the $q$-point quadrature integrates exactly a polynomial of the $(2 q-1)$ order (note that the two integration schemes coincide for the size ratio $\zeta=0$ ). The only exception is when the integration points in the nonlocal quadrature coincide. For instance, for two-point nonlocal integration and $\zeta=1$ the quadrature points coincide at $\hat{\eta}_{a 1,2}=0$; and for three-point nonlocal integration and $\zeta=0.5$ the three nonlocal quadrature points collapse to two points at $\hat{\eta}_{a 1,3}=\mp 0.5$, see (6)b. Furthermore, it can be shown (see Appendix A.1) that for $\zeta=0.5,1$ the two-point nonlocal quadrature is exact for any polynomial order. In this case, the two-point nonlocal quadrature scheme integrates exactly the fourth-order inertia terms $\rho^{\zeta} \mathbf{w}^{c T} \ddot{\mathbf{u}}{ }^{\zeta}$ in equation (5) for quadratic coarse-scale elements. For cases other than $\zeta=0.5,1$, a three-point nonlocal quadrature can be selected for quadratic coarse-scale elements to integrate the inertia term exactly, but that limits the maximum size ratio to $\zeta<0.5$. In Sections 3.4 and 4.2 we present the $\mathrm{C}^{2}$ dispersion curves computed using two-point and three-point nonlocal quadrature schemes for $0.5 \leq \zeta<1$ and $0<\zeta<0.5$, respectively. Similarly, it can be also shown that the three-point nonlocal quadrature for $\zeta=1 / 3$ is exact.

Remark 3. In equation (5), when microstructural features appear on the boundary, the non-local quadrature can be devised for integrals over the natural boundary $\partial \Omega^{t \zeta}$. See [36] for details.

Remark 4. The coarse-scale problem (5) can be further simplified by inserting a linear variation of the coarse-scale strain (2) and the test function gradient $\nabla \mathbf{w}^{c}$ into equation (5). For details, see $[35,36,37]$.

\subsection{The dynamic fine-scale problem}

The strong form of the fine-scale problem defined over the computational unit cell domain $\Theta_{a I}$ is given as

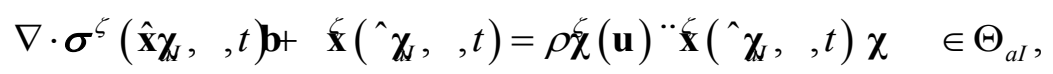

subjected to the following constraints $[35,36]$ 
a) $\mathbf{u}^{*}-$ periodic on $\partial \Theta_{a I}$,

b) $\int_{\partial \Theta_{a l}^{j}} \mathbf{u}^{*} d \gamma=\mathbf{0} \quad \forall j=1, n^{s} ; \quad \bigcup_{j=1}^{n^{s}} \partial \Theta_{a I}^{j}=\partial \Theta_{a I} ; \quad \partial \Theta_{a I}^{i} \cap \partial \Theta_{a I}^{j}=0 \quad \forall i \neq j$,

c) $\mathbf{u}^{\zeta}, \mathbf{t}^{\zeta}$ - continuous at the microconstituent interfaces.

Equation (8)b follows from the decomposition of the overall stress into a periodic $\boldsymbol{\sigma}^{\text {per }}\left(\hat{\mathbf{x}} \boldsymbol{\chi}_{\mathrm{s}}, t\right)$ and a non-periodic linear variation $\alpha\left(\hat{\mathbf{x}}_{a I}, t\right)$

$$
\boldsymbol{\sigma}^{\zeta}\left(\hat{\mathbf{x}} \chi_{I},, t\right)=\boldsymbol{\sigma}^{p e x}\left(\wedge^{\wedge} \chi_{I}, t\right)+\boldsymbol{\alpha}\left({ }^{\wedge}{ }_{a I}, \boldsymbol{t}\right) .
$$

For one-dimensional problems, $(8) \mathrm{a}$ and $(8) \mathrm{b}$ reduce to $u^{*}=0$ on $\partial \Theta_{a I}$ (see $[35,36]$ for details). Equation (8)c follows from the condition of perfectly bonded interfaces.

The weak form of the fine-scale problem follows from (3), by selecting periodic test function $\mathbf{W}=\mathbf{w}^{*}$ over the unit cell domain

$$
\begin{aligned}
& \int_{\Theta_{a I}}\left(\nabla \mathbf{w}^{*}\right)^{T} \sigma^{\zeta} d \Theta+\int_{\Theta_{a I}} \rho^{\zeta} \mathbf{w}^{* T} \ddot{\mathbf{u}}^{\zeta} d \Theta=\int_{\Theta_{a I}} \mathbf{w}^{* T} \mathbf{b}^{\zeta} d \Theta, \\
& \forall \mathbf{w}^{*} \in V^{*}=\left\{\mathbf{w}^{*} \in C^{0}\left(\Theta_{a I}\right), \mathbf{w}^{*} \text { - periodic on } \partial \Theta_{a I}, \int_{\partial \Theta_{a l}^{j}} \mathbf{w}^{*} d \gamma=\mathbf{0}\right\} .
\end{aligned}
$$

In equation (10), the traction term vanishes due to (9) and (8)a,b, as shown in [35].

Remark 5. In case of the long wave approximation, the last two terms in equation (10) are ignored, resulting in a quasi-static unit cell problem similar to that obtained from the $\mathrm{O}(1)$ homogenization theory.

\section{Analytical dispersion analysis}

The wave equation in elastic layered media has been considered by numerous investigators (see $[33,34,12,18,22]$, to mention just a few). The most common approach for analytical study is to consider the wave propagation in the Floquet-Bloch form, which is adopted hereafter for both the computational continua formulation (Section 3.1) and the analytical reference solution (Section 3.2). It provides the dispersion relation (Section 3.3) in the implicit closed form that relates frequency and a wave number. By this so-called analytical dispersion analysis, we solve the fine-scale problem in closed form (Section 3.1), whereas for the solution of the coarse-scale problem we assume that the coarse-scale solution is given in the Floquet-Bloch form. Thus we avoid solving the coupled system (5), (10) numerically. An alternative, numerical approach is pursued in Section 4.

The unit cell is assumed to consist of two phases denoted by $\Theta^{\beta}, \beta=1,2$, with the volume fractions denoted by $\varphi_{\beta}$, such that $\varphi_{1}+\varphi_{2}=1$. The Young's moduli and the densities are denoted as $E_{\beta}$ and $\rho_{\beta}$, respectively, as shown in Fig. 2a. The unit cell representing the same microstructure can be selected in a number of ways. Fig. 2 depicts symmetric and non-symmetric unit cell configurations. For the Floquet-Bloch solution, we consider the non-symmetric case in Fig. 2a, while for the numerical examples in Section 4 we consider the symmetric case in Fig. $2 \mathrm{~b}$. 
Fig. 2. is here

The piece-wise constant material properties are defined as

$$
L^{\zeta}(\chi)=\left\{\begin{array}{l}
E_{1}, \\
E_{2},
\end{array} \quad \rho^{\zeta}(\chi)=\left\{\begin{array}{l}
\rho_{1}, \quad \chi \in \Theta^{1}=\left(-\frac{l}{2}, \chi_{\text {intf }}\right) \\
\rho_{2}, \chi \in \Theta^{2}=\left(\chi_{\text {int }}, \frac{l}{2}\right)
\end{array}\right.\right.
$$

where $\chi_{\text {inf }}=l\left(\varphi_{1}-\varphi_{2}\right) / 2$ denotes the position of interface between two phases.

\subsection{The Floquet-Bloch wave for the $C^{2}$ fine-scale model problem}

Consider a one-dimensional elastic fine-scale problem (7),(8) in the absence of body forces

$$
\begin{aligned}
& \sigma_{, x}^{\zeta}\left(\hat{x}_{a I}, \chi, t\right)=\rho^{\zeta}(\chi) \ddot{u}^{\zeta}\left(\hat{x}_{a I}, \chi, t\right), \\
& \sigma^{\zeta}\left(\hat{x}_{a I}, \chi, t\right)=L^{\zeta}(\chi) u_{, \chi}^{\zeta}\left(\hat{x}_{a I}, \chi, t\right), \\
& u^{*}=0 \text { on } \partial \Theta_{a I}, \\
& u^{\zeta}, t^{\zeta} \text { - continuous at interface, }
\end{aligned}
$$

where the comma denotes a spatial derivative. Note that for the dispersion analysis all external forces are zero, and there are no prescribed initial conditions.

We seek a closed-form solution of equation (11)a in phase $\beta$ by considering the following harmonic wave with constant overall angular frequency $\omega$

$$
\begin{aligned}
& u_{\beta}^{\zeta}\left(\hat{x}_{a I}, \chi, t\right)=U_{\beta}^{\zeta}\left(\hat{x}_{a I}, \chi\right) e^{i \omega t} ; \quad \beta=1,2, \\
& \text { or } \\
& u^{\zeta}\left(\hat{x}_{a I}, \chi, t\right)= \begin{cases}U_{1}^{\zeta}\left(\hat{x}_{a I}, \chi\right) e^{i \omega t}, & \chi \in \Theta^{1}=\left(-\frac{l}{2}, \varphi_{1} l-\frac{l}{2}\right) \\
U_{2}^{\zeta}\left(\hat{x}_{a I}, \chi\right) e^{i \omega t}, & \chi \in \Theta^{2}=\left(\varphi_{1} l-\frac{l}{2}, \frac{l}{2}\right)\end{cases}
\end{aligned}
$$

Note that wave (12) written in the Floquet-Bloch form $[29,34,12]$ is

$$
\begin{aligned}
& u_{\beta}^{\zeta}\left(\hat{x}_{a I}, \chi, t\right)=U_{\beta}^{\zeta}\left(\hat{x}_{a I}, \chi\right) e^{i\left(k\left(\hat{x}_{a l}+\chi\right)+\omega t\right)}, \\
& U_{\beta}^{\zeta}=U_{\beta}^{\zeta} e^{-i k\left(\hat{x}_{a l}+\chi\right)},
\end{aligned}
$$

where $U_{\beta}^{\zeta}\left(\hat{x}_{a l}, \chi\right)$ is a periodic function and $k$ is a wave number (or Bloch parameter). The wave expression in (12)a is equivalent to (13) and is chosen here for convenience.

The coarse-scale and the perturbation displacement components in equation (2) are given by

$$
\begin{aligned}
& u^{c}\left(\hat{x}_{a I}, \chi, t\right)=U^{c}\left(\hat{x}_{a I}, \chi\right) e^{i \omega t} ; u_{\beta}^{*}\left(\hat{x}_{a I}, \chi, t\right)=U_{\beta}^{*}\left(\hat{x}_{a I}, \chi\right) e^{i \omega t}, \\
& U_{\beta}^{\zeta}=U_{\beta}^{*}+U^{c} ; \quad \beta=1,2 .
\end{aligned}
$$

Note that the Floquet-Bloch solution itself is defined on a single scale, i.e. $x=\hat{x}_{a I}+\chi$ (see Section 3.2). The contribution of the microstructure in the Floquet-Bloch solution is expressed by the periodic function $U_{\beta}^{\zeta}$, while in the dispersive $C^{2}$ model we introduce an explicit decomposition of the displacement into a coarse-scale contribution $U^{c}$ and a fine-scale 
perturbation $U_{\beta}^{*}(14) \mathrm{b}$. Thus, the fine-scale perturbation $U_{\beta}^{*}$ can be expressed as $U_{\beta}^{*}=U_{\beta}^{\zeta} e^{i k\left(\hat{x}_{a l}+\chi\right)}-U^{c}$, which follows from (13)b and (14)b.

Inserting (12) into (11)a yields

$$
\begin{aligned}
& U_{\beta, \chi \chi}^{\zeta}+k_{\beta}^{2} U_{\beta}^{\zeta}=0 ; \quad \beta=1,2, \\
& k_{\beta}=\omega \sqrt{\rho_{\beta} / E_{\beta}},
\end{aligned}
$$

where $k_{\beta}$ is the wave number of the $\beta$-th phase. The constraints (11)c,d can be rewritten as

$$
\begin{aligned}
& U_{1}^{*}\left(\hat{x}_{a I},-\frac{l}{2}\right)=U_{2}^{*}\left(\hat{x}_{a I}, \frac{l}{2}\right)=0, \\
& U_{1}^{\zeta}\left(\hat{x}_{a I}, \chi_{\text {intf }}\right)=U_{2}^{\zeta}\left(\hat{x}_{a I}, \chi_{\text {intf }}\right), \\
& E_{1} U_{1, \chi}^{\zeta}\left(\hat{x}_{a I}, \chi_{\text {intf }}\right)=E_{2} U_{2, \chi}^{\zeta}\left(\hat{x}_{a I}, \chi_{\text {intf }}\right) .
\end{aligned}
$$

It is well-known that the general solution of the wave equation (15) can be conveniently written as

$$
U_{\beta}^{\zeta}\left(\hat{x}_{a I}, \chi\right)=\hat{A}_{\beta}\left(\hat{x}_{a I}\right) e^{i k_{\beta} \chi}+\hat{B}_{\beta}\left(\hat{x}_{a I}\right) e^{-i k_{\beta} \chi} .
$$

The coefficients $\hat{A}_{\beta}\left(\hat{x}_{a I}\right)$ and $\hat{B}_{\beta}\left(\hat{x}_{a I}\right)$ are computed by inserting (17) into (16) and utilizing (14)b. This results in an algebraic system of equations for a given values of $U^{c}$ and $\Theta_{a I}$ with impedance $z_{\beta}=E_{\beta} k_{\beta}$ for the $\beta$-th phase

$$
\left[\begin{array}{cccc}
e^{-i k_{1} l / 2} & e^{i k_{1} l / 2} & 0 & 0 \\
0 & 0 & e^{i k_{2} l / 2} & e^{-i k_{2} l / 2} \\
e^{i k_{1} \chi_{\text {inf }}} & e^{-i k_{1} \chi_{\text {inf }}} & -e^{i k_{2} \chi_{\text {inf }}} & -e^{-i k_{2} \chi_{\text {inf }}} \\
z_{1} e^{i k_{1} \chi_{\text {inf }}} & -z_{1} e^{-i k_{1} \chi_{\text {inf }}} & -z_{2} e^{i k_{2} \chi_{\text {inf }}} & z_{2} e^{-i k_{2} \chi_{\text {inf }}}
\end{array}\right]\left[\begin{array}{c}
\hat{A}_{1}\left(\hat{x}_{a I}\right) \\
\hat{B}_{1}\left(\hat{x}_{a I}\right) \\
\hat{A}_{2}\left(\hat{x}_{a I}\right) \\
\hat{B}_{2}\left(\hat{x}_{a I}\right)
\end{array}\right]=\left[\begin{array}{c}
U^{c}\left(\hat{x}_{a I},-\frac{l}{2}\right) \\
U^{c}\left(\hat{x}_{a I}, \frac{l}{2}\right) \\
0 \\
0
\end{array}\right] .
$$

Remark 6. It can be shown that the resulting solution (17) satisfies the aforementioned hypothesis of an additive stress decomposition (9), $\sigma^{\zeta}=\sigma^{\text {per }}+\alpha \chi$, where

$$
\alpha\left(\hat{x}_{a I}, t\right)=\frac{1}{l}\left(E_{2} U_{2, \chi}^{\zeta}\left(\hat{x}_{a I}, \frac{l}{2}\right)-E_{1} U_{1, \chi}^{\zeta}\left(\hat{x}_{a I},-\frac{l}{2}\right)\right) e^{i \omega t} .
$$

\subsection{The reference solution}

In this section, we recall the general solution in the Floquet or Bloch form with the corresponding constraints [29], which yields the classical dispersion relation [30]. We consider the governing equation (11)a in absence of all external forces and employ a single coordinate system $x$ defined over an infinite domain. The Floquet-Bloch approach yields the following solution $[12,34]$

$$
\begin{aligned}
& u_{\beta}^{\zeta}(x, t)=U_{\beta}^{\zeta}(x) e^{i(k x+\omega t)} ; \quad \beta=1,2, \\
& U_{\beta}^{\zeta}(x)=A_{\beta} e^{i\left(k_{\beta}-k\right) x}+B_{\beta} e^{-i\left(k_{\beta}+k\right) x},
\end{aligned}
$$


where $U_{\beta}^{\zeta}(x)$ is a periodic function. The periodicity of $U_{\beta}^{\zeta}(x)$ yields the so-called pseudo- or quasi-periodicity condition $[33,12]$ of the overall displacement that can be written in terms of $U_{\beta}^{\zeta}$ as

$$
U_{2}^{\zeta}(x+l)=U_{1}^{\zeta}(x) e^{i k l}
$$

This pseudo-periodicity, known as the Floquet-Bloch condition, can be written for stress as

$$
E_{2} U_{2, x}^{\zeta}(x+l)=E_{1} U_{1, x}^{\zeta}(x) e^{i k l} .
$$

For perfectly bonded interfaces, we have continuous overall displacements and stresses

$$
U_{1}^{\zeta}\left(x_{\text {intf }}\right)=U_{2}^{\zeta}\left(x_{\text {intf }}\right) ; \quad E_{1} U_{1, x}^{\zeta}\left(x_{\text {intf }}\right)=E_{2} U_{2, x}^{\zeta}\left(x_{\text {intf }}\right) .
$$

Placing the origin of the coordinate at one of the interfaces, i.e. $x_{\text {inff }}=0$, and writing the system of equations with the consideration of pseudo-periodicity (20), (21) and the perfectly bonded interfaces (22), yields the following homogeneous algebraic system of equations for the coefficients $A_{\beta}, B_{\beta}[34,12]$

$$
\left[\begin{array}{cccc}
e^{-i\left(k_{1}-k\right) \varphi_{1} l} & e^{i\left(k_{1}+k\right) \varphi_{1} l} & -e^{i\left(k_{2}-k\right) \varphi_{2} l} & -e^{-i\left(k_{2}+k\right) \varphi_{2} l} \\
z_{1} e^{-i\left(k_{1}-k\right) \varphi_{1} l} & -z_{1} e^{i\left(k_{1}+k\right) \varphi_{1} l} & -z_{2} e^{i\left(k_{2}-k\right) \varphi_{2} l} & z_{2} e^{-i\left(k_{2}+k\right) \varphi_{2} l} \\
1 & 1 & -1 & -1 \\
z_{1} & -z_{1} & -z_{2} & z_{2}
\end{array}\right]\left[\begin{array}{l}
A_{1} \\
B_{1} \\
A_{2} \\
B_{2}
\end{array}\right]=\left[\begin{array}{l}
0 \\
0 \\
0 \\
0
\end{array}\right] .
$$

Requiring the determinant of the system (23) to vanish yields the classical dispersion relation [30], which will be considered hereafter as a reference solution for the dispersion analysis

$$
\cos (k l)=\cos \left(k_{1} \varphi_{1} l\right) \cos \left(k_{2} \varphi_{2} l\right)-\frac{1}{2}\left(\frac{z_{1}}{z_{2}}+\frac{z_{2}}{z_{1}}\right) \sin \left(k_{1} \varphi_{1} l\right) \sin \left(k_{2} \varphi_{2} l\right) .
$$

Remark 7. It can be shown that the zero-periodicity condition (16)a in the computational continua formulation reduces to the pseudo-periodicity (or Floquet-Bloch) condition of displacements (20) if the coarse-scale displacement is given by

$$
U^{c}\left(\hat{x}_{a I}, \chi\right)=U_{0}\left(\hat{x}_{a I}\right) e^{i k \chi} \text {. }
$$

Indeed, the following equation, (16)a

yields

$$
U_{1}^{\zeta}\left(\hat{x}_{a I},-\frac{l}{2}\right)=U_{0}\left(\hat{x}_{a I}\right) e^{-i k l / 2} ; \quad U_{2}^{\zeta}\left(\hat{x}_{a I}, \frac{l}{2}\right)=U_{0}\left(\hat{x}_{a I}\right) e^{i k l / 2},
$$

$$
U_{2}^{\zeta}\left(\hat{x}_{a I}, \frac{l}{2}\right)=U_{1}^{\zeta}\left(\hat{x}_{a I},-\frac{l}{2}\right) e^{i k l} .
$$

Thus, we have shown that the dispersive $\mathrm{C}^{2}$ formulation satisfies the Floquet-Bloch condition for displacements, but it may or may not satisfy it for other fields.

Remark 8. The constraints in the $\mathrm{C}^{2}$ formulation (26), (16)b,c are similar to the constraints (20) and (22) used for the reference solution if the locations of computational unit cells are physically feasible, i.e., for $\zeta=0.5$ or $\zeta=1$ for a two-point integration as discussed in Remark 1. Note that the dispersive $\mathrm{C}^{2}$ formulation makes no assumption about the stress pseudo-periodicity (21). However, in Appendix A.3, we prove that the stress pseudo-periodicity condition is satisfied by the $\mathrm{C}^{2}$ formulation for $\zeta=0.5,1$. Therefore these cases give exact dispersion relations. 


\subsection{Analytical dispersion analysis of the dispersive $C^{2}$ formulation}

In the reference solution, the algebraic system of equations (23) is homogeneous. Thus, the condition of having a set of nontrivial reference solutions (i.e., a zero determinant) provides an additional equation from which the dispersion relation (24) follows. Unlike the reference solution, the algebraic system of equations (18) arising from the dispersive $\mathrm{C}^{2}$ formulation is non -homogeneous, with the right-hand side depending on the macroscopic solution. Therefore, for the computational continua the additional equation is provided by the discrete form of the coarsescale solution (5).

For the dispersion analysis, we assume that the coarse-scale displacement is prescribed in the form of a harmonic wave (25)

$$
\begin{aligned}
& u^{c}\left(\hat{x}_{a I}, \chi, t\right)=U^{c}\left(\hat{x}_{a I}, \chi\right) e^{i \omega t}=U_{0}\left(\hat{x}_{a I}\right) e^{i(k \chi+\omega t)}, \\
& U^{c}\left(\hat{x}_{a I}, \chi\right)=e^{i k\left(\hat{x}_{a l}+\chi\right)} ; \quad U_{0}\left(\hat{x}_{a I}\right)=e^{i k \hat{x}_{a I} .}
\end{aligned}
$$

The length of the wave is defined through $k$ by $\lambda=2 \pi / k$. Fig. 3a shows the imaginary part of the wave, $\operatorname{Im}\left[U^{c}\right]=\sin (2 \pi x / \lambda)$, over the composite domain. Fig. $3 \mathrm{~b}$ compares different meshes of the coarse-scale domain for a fixed composite domain consisting of $n^{u c}$ unit cells, i.e., $\left|\Omega^{c}\right|=n^{u c} l$. For a coarse-scale mesh of $n^{e l}$ elements, $\left|\Omega^{c}\right|=n^{e l} L^{e l}$, the unit cell size ratio is given by $\zeta=l / L^{e l}=n^{e l} / n^{u c}$. Note that $n^{e l}$ is proportional to the size ratio $\zeta$ and therefore for a fixed $n^{u c}$, a better wave resolution is obtained with an increase in $\zeta$.

Fig. 3. is here

Following the Galerkin approximation, the test function $w^{c}$ is taken from the same set of functions as the trial solution $u^{c}$ in (27), subjected to a homogeneous boundary condition on $\partial \Omega^{c}$.

$$
\begin{aligned}
& w^{c}\left(\hat{x}_{a I}, \chi, t\right)=W^{c}\left(\hat{x}_{a I}, \chi\right) e^{i \omega t} \\
& W^{c}\left(\hat{x}_{a I}, \chi\right)=\sin \left(k\left(\hat{x}_{a I}+\chi\right)\right)
\end{aligned}
$$

For simplicity, we omit the constant amplitude in (27)b and (28)b, since it does not contribute to the final dispersion equation.

The dispersion relation $F(k, \omega)=0$ follows from the discrete solution of the coarse-scale problem (5) in the absence of body forces and prescribed tractions. Following the second order computational continua formulation, we consider a uniform mesh of quadratic coarse-scale elements (6), i.e., $J=J_{a I}$.

Recalling equation (5)

$$
J \sum_{a=1}^{n^{a^{l}}} \sum_{I=1}^{n^{i p}}\left\langle w_{,}^{c} \sigma^{\zeta}+w^{c} \rho^{\zeta} \ddot{u}^{\zeta}\right\rangle_{\Theta_{a l}}=0,
$$

and then inserting (12)a, (28) into (29) and considering $e^{i \omega t} \neq 0$, yields

$$
\sum_{a=1}^{n^{e l}} \sum_{I=1}^{n^{i p}} \sum_{\beta=1}^{2} v_{a l} \varphi_{\beta} E_{\beta}\left\langle W_{, \chi}^{c} U_{\beta, \chi}^{\zeta}-k_{\beta}^{2} W^{c} U_{\beta}^{\zeta}\right\rangle_{\Theta_{a l}^{\beta}}=0 .
$$


In the computational continua formulation, the exponential assumptions (27)b for $U^{c}$ and sinusoidal (28)b for $W^{c}$ are approximated by the quadratic functions $\tilde{U}^{c}$ and $\tilde{W}^{c}$, respectively, using three-node quadratic coarse-scale elements, as

$$
\begin{aligned}
& \tilde{U}^{c}\left(\hat{\eta}_{a I}, r\right)=\sum_{\delta=1}^{3} N_{\delta}^{c}\left(\hat{\eta}_{a I}+r\right) d_{a \delta}^{U^{c}} ; \quad \tilde{W}^{c}\left(\hat{\eta}_{a I}, r\right)=\sum_{\delta=1}^{3} N_{\delta}^{c}\left(\hat{\eta}_{a I}+r\right) d_{a \delta}^{W^{c}}, \\
& N_{1}^{c}(\eta)=\frac{1}{2} \eta(\eta-1) ; \quad N_{2}^{c}(\eta)=1-\eta^{2} ; \quad N_{3}^{c}(\eta)=\frac{1}{2} \eta(\eta+1),
\end{aligned}
$$

where $d_{a \delta}^{U^{c}}, d_{a \delta}^{W^{c}}$ are nodal values of the coarse-scale trial and test functions (27) and (28), respectively, in the $a$-th element; and $N_{\delta}^{c}$ is the quadratic-shape functions of $\eta=\hat{\eta}_{a I}+r$ (see Fig. 1 for definitions of $\eta$ and $r$ ).

Inserting the quadratic approximation (31) into (30) yields the dispersion relation

$$
F(k, \omega) \equiv \sum_{a=1}^{n^{e l}} \sum_{I=1}^{n^{i p}} \sum_{\beta=1}^{2} v_{a I} \varphi_{\beta} E_{\beta}\left\langle\tilde{W}_{, r}^{c}\left(\hat{\eta}_{a I}, r\right) \tilde{U}_{\beta, r}^{\zeta}\left(\hat{\eta}_{a I}, r\right)-k_{\beta}^{2} \tilde{W}^{c}\left(\hat{\eta}_{a I}, r\right) \tilde{U}_{\beta}^{\zeta}\left(\hat{\eta}_{a I}, r\right)\right\rangle_{\Theta_{a l}^{\prime \beta}}=0,
$$

where $\Theta_{a I}^{\prime \beta}=\Theta_{a I}^{\beta} / J$, and $\tilde{U}_{\beta}^{\zeta}$ is an overall displacement approximation (17) resulting from the coarse-scale displacement $\tilde{U}^{c}$ in the right-hand side of (18).

Note that the strain gradient introduced in the $\mathrm{C}^{2}$ formulation by (2) contributes to (32) implicitly, through the quadratic approximation $\tilde{W}^{c}$ and $\tilde{U}^{c}$, where the latter is used to compute $\tilde{U}_{\beta}^{\zeta}$ from (18). The dependence of $F$ on $(k, \omega)$ is implicit. The frequency $\omega$ appears in (32) through $k_{\beta}$, defined in (15), and through $z_{\beta}=E_{\beta} k_{\beta}$, involved in the computation of $\tilde{U}_{\beta}^{\zeta}$. The wave number $k=2 \pi / \lambda$ appears in (32) implicitly, through an approximation of the coarse-scale displacement $U^{c}$ in (27). For a detailed implementation of the dispersion relation (32) see Appendix A.2.

\subsection{Dispersion curves for the dispersive $C^{2}$ formulation}

In this section, we study the dispersion relation (32) and build the dispersion diagrams for different unit cell sizes reflected by the size ratio $\zeta=l / L^{e l}$. We consider a periodic heterogeneous medium with the following geometric and material parameters (Fig. 2a)

$$
\varphi_{1}=0.5 ; \quad E_{1}=100 ; \quad E_{2}=1 ; \quad \rho_{1}=3 ; \quad \rho_{2}=1 .
$$

All results are given with respect to the normalized frequency $\bar{\omega}$ and the wave number $\bar{k}$ defined as

$$
\begin{aligned}
& \bar{\omega}=\frac{\omega l}{2 \pi} \sqrt{\frac{\rho^{c}}{E^{c}}}=\frac{\omega l}{\omega_{0} \lambda} ; \quad \bar{k}=\frac{k l}{2 \pi}=\frac{l}{\lambda}, \\
& \omega_{0}=k \sqrt{E^{c} / \rho^{c}} ; E^{c}=\frac{E_{1} E_{2}}{\varphi_{1} E_{2}+\varphi_{2} E_{1}} ; \rho^{c}=\varphi_{1} \rho_{1}+\varphi_{2} \rho_{2} .
\end{aligned}
$$

For comparison, we also consider the dispersion relation of the $\mathrm{O}(1)$ micro-inertia formulation [24], in the normalized form 


$$
\bar{\omega}=\frac{\bar{k}}{\sqrt{1+\bar{k}^{2} \bar{D}^{c}}} ; \quad \bar{D}^{c}=\frac{1}{3}\left[\frac{\pi \varphi_{1} \varphi_{2}\left(E_{1} \rho_{1}-E_{2} \rho_{2}\right)}{\rho^{c}\left(\varphi_{2} E_{1}+\varphi_{1} E_{2}\right)}\right]^{2} .
$$

Note that the nondispersive formulation does not account for the dispersion effect; that is, the wave travels at a constant speed $\omega / k$. For example, the classical $\mathrm{O}(1)$ homogenization (not the $\mathrm{O}(1)$ micro-inertia) is nondispersive: the dispersion relation is linear, resulting in the constant wave speed

$$
\omega=\omega_{0}=k \sqrt{E^{c} / \rho^{c}} .
$$

In the dispersive $\mathrm{C}^{2}$ formulation, the equation $F(k, \omega)=0$ is complex, while the frequency $\omega$ and the wave number $k$ are real. Equation (32) is solved numerically (in Matlab) for $\omega$ and given the discrete value of $k$. Here, $k$ is associated with the discrete set of wavelengths $\lambda=2\left|\Omega^{c}\right| / p$, where $p \in \square$ and $1 \leq p \leq n^{u c}$ are employed to satisfy $\left.W^{c}\right|_{\partial \Omega^{c}}=0$. The normalized wave number (34) becomes $\bar{k}=p /\left(2 n^{u c}\right)$. In the implementation, we consider domains with a large number of unit cells as representing a wide range of discrete values of $\bar{k}$. We use two-point integration for $0.5 \leq \zeta \leq 1$ and three-point integration for cases where $\zeta<0.5$ (see Remark 2).

Fig. $4 \mathrm{a}$ depicts the dispersion diagrams obtained from the dispersion relations of the nondispersive formulation (36), the $\mathrm{O}(1)$ micro-inertia (35), the dispersive $C^{2}(32)$, and the reference solution (24) for the two cases of $\zeta=0.5,1$. It can be seen that the dispersive $\mathrm{C}^{2}$ curve coincides with the reference solution (24). Note that for $\zeta=0.5,1$ the dispersive $\mathrm{C}^{2}$ solution of the Floquet-Bloch type is equivalent to the reference solution (see Remark 8 and Appendix A.3) for a wide range of frequencies. This will be further discussed in Section 4.3.

Fig. $4 \mathrm{~b}$ depicts the dispersive $\mathrm{C}^{2}$ solutions for $\zeta=1 / 4,1 / 3,2 / 3,3 / 4$. It can be seen that for unit cell sizes other than $\zeta=0.5,1$, the dispersive $\mathrm{C}^{2}$ results are in good agreement with the reference solution up to $\bar{k} \leq \zeta$ (shown for $\zeta<0.5$ ), which refers to the physical limit of a wavelength being larger than the coarse-scale element size $\bar{k}=l / \lambda \leq \zeta L^{e l} / L^{e l}=\zeta$. Note that the smaller size ratio $\zeta$ is the lower limit. For $\zeta \rightarrow 0$ the $C^{2}$ formulation becomes nondispersive, identical to that of $\mathrm{O}(1)$ homogenization. The upshot of this observation is that for a given microstructural size, the accuracy of the $\mathrm{C}^{2}$ formulation improves with $\zeta \rightarrow 1$, with the only exception being around $\zeta=0.5$ (for quadratic coarse-scale elements).

\section{Fig. 4. is here}

For comparison, we also study the $C^{2}$ dispersion curves (32) for $\zeta=1 / 2,3 / 4,1$ obtained with the classical Gauss quadrature points (corresponding to $\zeta=0$ in (6)) rather with the nonlocal quadrature (6). For this example, we employ a three-point Gauss quadrature to integrate exactly the inertia term. Fig. 5 shows that the results obtained with the classical Gauss quadrature are worse than those obtained with nonlocal quadrature, in particular by increasing the size ratio $\zeta \rightarrow 1$ (Fig. 4). We will further refine this matter in Section 4.1.

Fig. 5. is here 


\section{Numerical dispersion analysis of the $\mathrm{C}^{2}$ formulation and numerical verification}

In this section, we consider the discrete form of the dispersive $\mathrm{C}^{2}$ formulation in the absence of body forces. In Section 4.1, we derive the coupled discrete fine-coarse-scale system of equations. In Section 4.2, we introduce the modal analysis for the discrete system of equations and compute numerical values for the dispersion diagram; and in Section 4.3, we present an example with the stop and pass bands. Finally, in Section 4.4, we consider a numerical example of the wave propagation problem to support the findings of the dispersion analysis.

\subsection{Numerical discretization of the coupled system}

The two-scale problem is two-way coupled, and thus, requires simultaneous solution of the coarse and fine-scale problems, (5) and (10). The trial and test functions are discretized as

$$
\begin{aligned}
& \mathbf{u}^{*}\left(\hat{\mathbf{x}}_{a I}, \mathbf{N}\right)=\boldsymbol{\chi} \mathbf{d}\left({ }^{*}{ }^{*}\left({ }^{\wedge} \mathbf{w}\right) ; \mathbf{x}^{*}\left(\hat{\boldsymbol{X}}_{a I}, \mathbf{N}\right)=\boldsymbol{\chi} \mathfrak{k}(\mathbf{x}){ }^{*}\left({ }_{a I}{ }_{a I}\right),\right. \\
& \mathbf{u}^{c}\left(\hat{\mathbf{x}}_{a I}, \mathbf{N}\right)=\mathbf{x}{ }^{c}(\hat{X} \mathbf{d},) \mathbf{w}^{c} ; \mathbf{x}^{c}\left(\hat{X}_{a I}, \mathbf{N}\right)=\mathbf{x}{ }^{c}\left(\chi^{c} \mathfrak{q},\right)^{c} \text {, }
\end{aligned}
$$

where $\mathbf{N}^{f}, \mathbf{N}^{c}$ are the fine- and coarse-scale shape functions, respectively; $\mathbf{d}^{*}$ and $\mathbf{d}^{c}$ are the discrete fine-scale and coarse-scale displacements, respectively; $\mathbf{c}^{*}$ and $\mathbf{c}^{c}$ are corresponding nodal values of the test functions.

The discrete trial and test functions of the fine-scale problem $\mathbf{d}^{*}, \mathbf{c}^{*}$ have dependent degreesof-freedom due to periodicity and normalization conditions (8)a,b. The fine-scale independent degrees-of-freedom, $\tilde{\mathbf{d}}^{*}$ and $\tilde{\mathbf{c}}^{*}$, can be defined through the transformation matrix $\mathbf{T}$ by

$$
\mathbf{d}^{*}=\mathbf{T} \tilde{\mathbf{d}}^{*} ; \quad \mathbf{c}^{*}=\mathbf{T} \tilde{\mathbf{c}}^{*} \text {. }
$$

The coupled discrete system is obtained by inserting interpolants (37) and constraints (38) into the corresponding weak forms (5), (10) and requiring arbitrariness of independent degrees-offreedom $\mathbf{c}^{\mathfrak{c}}, \tilde{\mathbf{c}}^{*}$. The resulting discrete form is given by

$$
\begin{gathered}
\mathbf{K d}+\mathbf{M} \ddot{\mathbf{d}}=\mathbf{F}^{\text {ext }}, \\
\mathbf{d}=\mathbf{d}_{0} \text { at } t=0 ; \quad \mathbf{d}=\overline{\mathbf{d}} \text { on } \partial \Omega^{u \zeta} ; \mathbf{t}^{\zeta}=\overline{\mathbf{t}} \text { on } \partial \Omega^{t \zeta},
\end{gathered}
$$

where

$$
\mathbf{d}=\left[\begin{array}{c}
\mathbf{d}^{c} \\
\tilde{\mathbf{d}}^{*}
\end{array}\right] ; \quad \mathbf{K}=\left[\begin{array}{cc}
\mathbf{K}^{c} & \mathbf{K}^{c f} \\
\tilde{\mathbf{K}}^{f c} & \tilde{\mathbf{K}}^{f}
\end{array}\right] ; \quad \mathbf{M}=\left[\begin{array}{cc}
\mathbf{M}^{c} & \mathbf{M}^{c f} \\
\tilde{\mathbf{M}}^{f c} & \tilde{\mathbf{M}}^{f}
\end{array}\right] ; \quad \mathbf{F}^{e x t}=\left[\begin{array}{c}
\mathbf{F}^{c} \\
\mathbf{F}^{f}
\end{array}\right],
$$

and

$$
\begin{aligned}
& \mathbf{K}^{c}=\sum_{a=1}^{n^{e l}} \sum_{I=1}^{n^{i p}} v_{a I} J_{a I}\left\langle\mathbf{B}^{c T} \mathbf{L}^{\zeta} \mathbf{B}^{c}\right\rangle_{\Theta_{a l}} ; \quad \mathbf{K}^{c f}=\sum_{a=1}^{n^{e l}} \sum_{I=1}^{n^{i p}} v_{a I} J_{a I}\left\langle\mathbf{B}^{c T} \mathbf{L}^{\zeta} \mathbf{B}^{f}\right\rangle_{\Theta_{a l}}, \\
& \mathbf{K}^{f}=\int_{\Theta_{a l}} \mathbf{B}^{f T} \mathbf{L}^{\zeta} \mathbf{B}^{f} d \Theta ; \quad \mathbf{K}^{f c}=\int_{\Theta_{a l}} \mathbf{B}^{f T} \mathbf{L}^{\zeta} \mathbf{B}^{c} d \Theta, \\
& \mathbf{M}^{c}=\sum_{a=1}^{n^{l l}} \sum_{I=1}^{n^{i p}} v_{a I} J_{a I}\left\langle\mathbf{N}^{c T} \rho^{\zeta} \mathbf{N}^{c}\right\rangle_{\Theta_{a l}} ; \quad \mathbf{M}^{c f}=\sum_{a=1}^{n^{n l}} \sum_{I=1}^{n^{i p}} v_{a I} J_{a I}\left\langle\mathbf{N}^{c T} \rho^{\zeta} \mathbf{N}^{f}\right\rangle_{\Theta_{a l}}, \\
& \mathbf{M}^{f}=\int_{\Theta_{a l}} \mathbf{N}^{f T} \rho^{\zeta} \mathbf{N}^{f} d \Theta ; \quad \mathbf{M}^{f c}=\int_{\Theta_{a l}} \mathbf{N}^{f T} \rho^{\zeta} \mathbf{N}^{c} d \Theta, \\
& \mathbf{F}^{c}=\int_{\partial \Omega^{\zeta \zeta}} \mathbf{N}^{c T} \mathbf{t}^{\zeta} d \Gamma+\sum_{a=1}^{n^{e l}} \sum_{I=1}^{n^{i p}} v_{a I} J_{a I}\left\langle\mathbf{N}^{c T} \mathbf{b}^{\zeta}\right\rangle_{\Theta_{a l}} ; \mathbf{F}^{f}=\int_{\Theta_{a l}} \mathbf{N}^{f T} \mathbf{b}^{\zeta} d \Theta,
\end{aligned}
$$

with 


$$
\begin{aligned}
\tilde{\mathbf{K}}^{f} & =\mathbf{T}^{T} \mathbf{K}^{f} \mathbf{T} ; \quad \tilde{\mathbf{K}}^{f c}=\mathbf{T}^{T} \mathbf{K}^{f c} ; \quad \tilde{\mathbf{F}}^{f}=\mathbf{T}^{T} \mathbf{F}^{f}, \\
\tilde{\mathbf{M}}^{f} & =\mathbf{T}^{T} \mathbf{M}^{f} \mathbf{T} ; \quad \tilde{\mathbf{M}}^{f c}=\mathbf{T}^{T} \mathbf{M}^{f c} .
\end{aligned}
$$

In the above, $\mathbf{B}^{c}$ and $\mathbf{B}^{f}$ are symmetric gradients of the shape functions, $\mathbf{N}^{c}$ and $\mathbf{N}^{f}$, respectively. For semi-discretization in time, the Newmark algorithm is employed.

Remark 10. For periodic meshes, periodicity can be alternatively enforced by assigning the same node number to the periodic nodes. The normalization condition (8)b can be enforced using Lagrange multipliers instead of the transformation matrix $\mathbf{T}$ approach in (38).

\subsection{Modal analysis}

Dynamic properties of the periodic heterogeneous media can be also studied by modal analysis. The numerical dispersion analysis is based on the assumption that fine- and coarsescale displacements are in the form of harmonic wave

$$
\begin{aligned}
& u^{\zeta}\left(\hat{x}_{a I}, \chi, t\right)=U^{\zeta}\left(\hat{x}_{a I}, \chi\right) e^{i \omega t} \\
& u^{c}\left(\hat{x}_{a I}, \chi, t\right)=U^{c}\left(\hat{x}_{a I}, \chi\right) e^{i \omega t} \\
& u^{*}\left(\hat{x}_{a I}, \chi, t\right)=U^{*}\left(\hat{x}_{a I}, \chi\right) e^{i \omega t} \\
& u^{\zeta}=u^{c}+u^{*}
\end{aligned}
$$

Assuming that the test functions are in the same space as the trial solution (40), and inserting the trial and test functions into the coupled system of equations, (29) and (10), and since $e^{i \omega t} \neq 0$ yields

$$
\begin{gathered}
\sum_{a=1}^{n^{e l}} \sum_{I=1}^{n^{i p}}\left\langle W_{, \chi}^{c} L^{\zeta} U_{, \chi}^{\zeta}-\omega^{2} W^{c} \rho^{\zeta} U^{\zeta}\right\rangle_{\Theta_{a I}}=0 \\
\int_{\Theta_{a l}}\left(W_{, \chi}^{*} L^{\zeta} U_{, \chi}^{\zeta}-\omega^{2} W^{*} \rho^{\zeta} U^{\zeta}\right) d \Theta=0
\end{gathered}
$$

Note that in principal the process yielding (41) is similar to the analytical dispersion analysis described in Section 3, which utilized similar expansions, (12) and (14). The main difference is that the coarse-scale displacement $U^{c}$ here is not expressed in a specific spatial form as in (27). Furthermore, the fine-scale problem is not solved analytically but rather numerically as a part of the discrete coupled problem (39).

The system (41), discretized based on (39), results in an eigenvalue problem with eigenvalues $\omega^{2}$ and eigenvectors $\mathbf{d}^{U}=e^{i \omega t} \mathbf{d}$

$$
\left(\mathbf{K}-\omega^{2} \mathbf{M}\right) \mathbf{d}^{U}=\mathbf{0}
$$

The eigenvalue analysis of (42) provides the frequency modes of the discretized problem. The number of modes is equal to the total number of nodes $n^{n}$ used in both fine- and coarsescale problems. We consider 30 quadratic coarse-scale elements and 9 quadratic elements per unit cell; the number of unit cells per element is controlled by the size ratio $\zeta$. The frequency mode $\omega_{p}$ is related to the wavelength $\lambda_{p}=2\left|\Omega^{c}\right| / p$ and the wave number $k_{p}=2 \pi / \lambda_{p}$ through the indices $p=1, \ldots n^{n}$. The solution of the eigenvalue problem (42) provides the numerical values for the dispersion diagram $\left(k_{p}, \omega_{p}\right)$. In the present study, we consider a symmetric unit cell configuration, as shown in Fig. $2 \mathrm{~b}$. 
Fig. 6a shows the dispersion diagrams obtained from the modal analysis of the dispersive $\mathrm{C}^{2}$ formulation for various $\zeta$ and compares these results with the reference dispersion curve (24). It can be seen that the results are consistent with the analytical diagrams presented earlier in Fig. 4. On the other hand, if instead of a nonlocal quadrature, the classical Gauss quadrature is employed in conjunction with the $\mathrm{C}^{2}$ modal analysis, the resulting dispersion curves are those shown in Fig. 6b. The importance of the nonlocal quadrature can be clearly observed by comparing Figs. $7 \mathrm{a}$ and $7 \mathrm{~b}$.

Fig. 6. is here

\subsection{Stop and pass frequency bands}

In previous sections, we compared various dispersion curves mostly related to the first frequency branch. In the present subsection, we extend the frequency and wave numbers range in a dispersion diagram in order to demonstrate the so-called pass and stop bands, i.e. the frequency ranges with and without solutions, respectively. Within a stop band, no wave propagation takes place. Thus, an observation of stop and pass bands is important for predicting the vibrations of composite materials and can be applied to electric and acoustic filters, noise control devices, ultrasonic transducers, crystal lattices, etc. [12,33,34].

Fig. 7 depicts the dispersion diagrams for the dispersive $C^{2}$ formulation, obtained by the modal analysis for $\zeta=0.5$, and the Floquet-Bloch solution (24) for a wide range of frequency modes. The stop bands are indicated by grey areas, while clear areas are related to the pass bands. The stop and pass frequency bands are the same for the dispersive $\mathrm{C}^{2}$ solution for $\zeta=0.5$ and the reference solution. The dispersive $\mathrm{C}^{2}$ diagram in Fig. 8 shows only one branch within every pass frequency band, thus providing for the solution's uniqueness. The choice of the branch follows from sorting the wave numbers as $k_{p}=2 \pi / \lambda_{p}$.

Fig. 7. is here

\subsection{Numerical example of an initial boundary value problem}

To compare the dynamic behavior of the dispersive $C^{2}$ solution with the direct numerical simulation (DNS) and the $\mathrm{O}(1)$ micro-inertia approach [24], we consider a wave propagation problem in a periodic one-dimensional heterogeneous medium. We consider the composite rod with 50 unit cells in a symmetric configuration (Fig. 2b) and with the material parameter listed in (33). The dispersive $C^{2}$ solution is computed for the case when the coarse-scale element is equal to the unit cell size, i.e. $\zeta=1$. We use 50 quadratic coarse-scale elements and 9 quadratic finescale elements per unit cell. The DNS mesh consists of $50 \times 9$ quadratic elements.

The wave excitation is formed by applying a sinusoidal high frequency $\omega_{\max }$ with displacement at the left end of the rod. The right end of the rod is held free. The frequency $\omega_{\max }$ corresponds to the highest normalized frequency $\bar{\omega}_{\max } \approx 0.346$ of the first branch (see Fig. 6a), corresponding to $\bar{k}=0.5$ with $\lambda=2 l=2 L^{e l}$. Thus, the initial boundary condition is defined as

$$
\left.u\right|_{\partial \Omega_{l e f t}^{c}}=\sin \left(\omega_{\max } t\right) ; \quad \omega_{\max }=\bar{\omega}_{\max } \frac{2 \pi}{l} \sqrt{\frac{E^{c}}{\rho^{c}}} .
$$


Fig. 8 depicts the displacement distribution at two different time instances: prior to the wave reflection and after. The coarse-scale displacement is shown for the dispersive $\mathrm{C}^{2}$ and $\mathrm{O}(1)$ micro-inertia solutions, while the total displacement is plotted for DNS. It can be seen that for this very high frequency regime the results for the dispersive $C^{2}$ formulation agree well with the DNS and clearly outperform the $\mathrm{O}(1)$ micro-inertia approach, which is a dispersive solution that assumes the steady-state unit cell problem.

Fig. 8 is here

Fig.9 depicts the displacement error in space for the dispersive $C^{2}$ solution and the $\mathrm{O}(1)$ micro-inertia with respect to the DNS solution. The relative error $\bar{e}_{L_{2}}^{T}(x)$ is defined as

$$
\bar{e}_{L_{2}}^{T}(x)=\frac{\sqrt{\int_{T}\left[u(x, t)-u_{\mathrm{DNS}}(x, t)\right]^{2} d} t}{\sqrt{\int_{T}\left[u_{\mathrm{DNS}}(x, t)\right]^{2} d t}} 100
$$

Fig. 9 is here

The computational cost of the method has been estimated by counting the number of floating point operations (FLOPS) for solving the discrete system of algebraic equations at $m$ number of time steps. For the dispersive $\mathrm{C}^{2}$ method, the block system of equation (39) is solved by using a staggered time-integration scheme based on the Newmark algorithm. We considered a onedimensional periodic elastic bar with the size ratio $\zeta=1$.

Assuming that the number of independent degrees of freedom at a coarse scale $n_{c}$ is much large than at a fine scale $n_{f}$, i.e., $n_{c} \square n_{f}$, and without taking advantage of sparsity, the computational cost for the dispersive $\mathrm{C}^{2}$ method $\left(\tau_{C^{2}}\right), \mathrm{O}(1)$ micro-inertia $\left(\tau_{\mathrm{mi}}\right)$, and direct numerical simulation ( $\tau_{D N S}$ ) at a finite number of steps $m$ are as follows

$$
\tau_{C^{2}} \square O\left(n_{c}^{3}\right) ; \tau_{\mathrm{mi}} \square O\left(n_{c}^{3}\right) ; \quad \tau_{D N S} \square O\left(n_{c}^{3} n_{f}^{3}\right) .
$$

In this case the order of computational cost for the elastic dispersive $\mathrm{C}^{2}$ method is the same as for the $\mathrm{O}(1)$ micro-inertia formulation and lower than that of the DNS by a factor of $n_{f}^{3}$.

For a very large number of time steps $m \square 1$ and assuming the fine- and coarse-scale degrees of freedom being of the same order, $n_{c} \square n_{f} \square n$, the computational cost of the time loops is estimated as

$$
\tau_{C^{2}} \square O\left(m n^{3}\right) ; \quad \tau_{\mathrm{mi}} \square O\left(m n^{2}\right) ; \quad \tau_{D N S} \square O\left(m n^{4}\right)
$$

Here the cost of the dispersive $C^{2}$ approach is lower than that of DNS, but higher than of the $\mathrm{O}$ (1) micro-inertia formulation by a factor of $n$, since the unit cell problem has to be solved for multiple right-hand sides equal to the number of unit cells. Finally, for inelastic problems, the computational cost of the micro-inertia and dispersive $\mathrm{C}^{2}$ formulations are of the same order.

For a detailed analysis of the computational cost for the dispersive $\mathrm{C}^{2}$ method see [43]. 


\section{Conclusions and future research directions}

A dispersive variant of the computational continua $\left(\mathrm{C}^{2}\right)$ formulation has been developed. Based on the dispersion analysis, the dispersive $\mathrm{C}^{2}$ formulation featuring a nonlocal quadrature and the transient unit cell problem has been found to possess outstanding dispersion properties. The dispersion analysis has been conducted in a periodic elastic medium using two approaches: 1) the Floquet-Bloch wave solution, and 2) the modal analysis of the coupled discrete system. The dispersive curves were compared with the classical reference solution. Although the modal analysis is more general, the analytical Floquet-Bloch approach provides a valuable insight into the form of the dispersion relations. Thus, it has been found that there exist narrow regions around two superconvergent points corresponding to the unit cell size being either half of the coarse-scale element size or equal to it, in which case the dispersive $\mathrm{C}^{2}$ formulation practically coincides with the reference solution even for very high frequencies.

The striking accuracy of the method stems in part from its unique formulation, which is free of scale separation and accounts for microstructures of finite size by means of nonlocal quadrature. The importance of nonlocal quadrature has been demonstrated by replacing it with the classical Gauss quadrature. The dispersive $\mathrm{C}^{2}$ formulation has been verified for the wave propagation problem with a very short wavelength.

The present approach, however, is not without limitations, which are summarized below:

- The size of the coarse-scale element should be larger or equal to the unit cell size (unlike the method in [24]). This is the limitation of nonlocal quadrature.

- The dispersive $\mathrm{C}^{2}$ formulation requires a solution of the time-dependent problem at a fine-scale (unit cell) and therefore is computationally taxing in comparison to methods that consider the unit cell problem to be steady state.

Future work will focus on addressing the computational limitations of the dispersive $\mathrm{C}^{2}$ formulation, including but not limited to:

(a) Since the formulation gives rise to a transient unit cell problem, it is necessary to devise an efficient monolithic and staggered block-scheme to effectively solve the coupled equations (39), see [43];

(b) Extension to nonlinear problems remains computationally challenging. We shall explore employing residual-free deformation modes that a priori satisfy the unit cell equilibrium equations [35-37]; and

(c) While the method performed fairly well throughout the entire range of the size ratios, the most remarkable performance was observed in the vicinity of two super-convergent points. It remains to be seen to what extent such a superconvergent behavior extends to multiple dimensions.

\section{Acknowledgments}

The ONR Grant N00014-12-1-0558 to Columbia University is gratefully acknowledged. The authors are also grateful to the reviewers for their valuable comments, which helped in improving the manuscript.

\section{Appendix A}

\section{A.1. Exact integration}

The remainder between the exact integration and the nonlocal quadrature is given by 


$$
\begin{aligned}
R(f) \equiv I(f)-I^{\text {nonlocal }}(f) & =\int_{\Omega^{e \zeta}} f(x) d \Omega-\sum_{I=1}^{n^{i p}} v_{I} J \frac{1}{\left|\Theta_{I}\right|} \int_{\Theta_{I}} f\left(\hat{x}_{I}+\chi\right) d \chi \\
& =\int_{-1}^{1} J f(\eta) d \eta-\sum_{I=1}^{n^{i p}} \frac{v_{I}}{2 \zeta} \int_{-\zeta}^{\zeta} J f\left(\hat{\eta}_{I}+r\right) d r .
\end{aligned}
$$

Consider now an integrand of a polynomial of $(m-1)$-th order

$$
J f(\eta)=J f\left(\hat{\eta}_{k}+r\right)=\sum_{k=1}^{m} a_{k}\left(\hat{\eta}_{k}+r\right)^{k-1} \equiv P^{m-1}(\eta),
$$

with $a_{k}$ being polynomial coefficients.

The exact and the nonlocal integration of the polynomial give

$$
\begin{aligned}
& I\left(P^{m-1}\right)=\sum_{p=1}^{\operatorname{int}[(m+1) / 2]} \frac{2 a_{(2 p-1)}}{2 p-1}, \\
& I^{\text {nonlocal }}\left(P^{m-1}\right)=\sum_{k=1}^{m} \sum_{I=1}^{n^{i p}} \frac{v_{I} a_{k}}{2 \zeta k}\left[\left(\hat{\eta}_{I}+\zeta\right)^{k}-\left(\hat{\eta}_{I}-\zeta\right)^{k}\right] .
\end{aligned}
$$

Then the remainder in (43) is given as

$$
\begin{aligned}
R\left(P^{m-1}\right) & =\sum_{p=1}^{\operatorname{int}[(m+1) / 2]} \frac{a_{(2 p-1)}}{2 p-1}\left(2-\sum_{I=1}^{n^{i p}} \frac{v_{I}}{2 \zeta}\left[\left(\hat{\eta}_{I}+\zeta\right)^{(2 p-1)}-\left(\hat{\eta}_{I}-\zeta\right)^{(2 p-1)}\right]\right) \\
& -\sum_{p=1}^{\operatorname{int}[m / 2]} \sum_{I=1}^{n^{i p}} \frac{v_{I} a_{2 p}}{4 \zeta p}\left[\left(\hat{\eta}_{I}+\zeta\right)^{2 p}-\left(\hat{\eta}_{I}-\zeta\right)^{2 p}\right] .
\end{aligned}
$$

The two-point nonlocal quadrature (6)a for $\zeta=0.5$ and $\zeta=1$ has integration points at $\hat{\eta}_{1,2}=\mp 0.5$ and $\hat{\eta}_{1,2}=0$, respectively. It gives $\left(\hat{\eta}_{I} \pm \zeta\right)=0, \pm 1$, resulting in a zero remainder in equation (46). Thus for $\zeta=0.5,1$, the two-point nonlocal quadrature integrates exactly polynomials of any order. Similarly, it can be shown, that for the three-point integration (6)b, the case of $\zeta=1 / 3$ yields the exact integration of a polynomial of any order. In the aforementioned discussion integral over the unit cell domain, $\int_{\Theta_{I}} f\left(\hat{x}_{I}+\chi\right) d \chi$ was assumed to be computed exactly (45); otherwise the fine-scale approximation error may contribute to the error in the nonlocal quadrature.

\section{A.2. Discretization of the dispersion relation}

For the quadratic element $a$-th in (31), we may rewrite the coarse-scale trial and test functions as

$$
\begin{aligned}
& \tilde{U}^{c}(\eta)=a_{a}^{U} \eta^{2}+b_{a}^{U} \eta+c_{a}^{U} ; \quad \tilde{W}^{c}(\eta)=a_{a}^{W} \eta^{2}+b_{a}^{W} \eta+c_{a}^{W}, \\
& a_{a}^{U}=\frac{1}{2}\left(d_{a 1}^{U^{c}}+d_{a 3}^{U^{c}}\right)-d_{a 2}^{U^{c}} ; \quad b_{a}^{U}=\frac{1}{2}\left(d_{a 3}^{U^{c}}-d_{a 1}^{U^{c}}\right) ; \quad c_{a}^{U}=d_{a 2}^{U^{c}}, \\
& a_{a}^{W}=\frac{1}{2}\left(d_{a 1}^{W^{c}}+d_{a 3}^{W^{c}}\right)-d_{a 2}^{W^{c}} ; \quad b_{a}^{W}=\frac{1}{2}\left(d_{a 3}^{W^{c}}-d_{a 1}^{W^{c}}\right) ; \quad c_{a}^{W}=d_{a 2}^{W^{c}} .
\end{aligned}
$$


The nodal values are defined by computing the values of $U^{c}, W^{c}$ in (27), (28) at nodal coordinates $x_{a \delta}$

$$
d_{a \delta}^{U^{c}}=U^{c}\left(x_{a \delta}\right)=e^{i k x_{a \delta}} ; \quad d_{a \delta}^{W^{c}}=W^{c}\left(x_{a \delta}\right)=\sin \left(k x_{a \delta}\right) ; \quad x_{a \delta}=\frac{L^{e l}}{2}(2 a+\delta-3) .
$$

Substituting $\eta=\hat{\eta}_{a I}+r$ into (47) yields a quadratic approximation expressed in local coordinate $r$

$$
\begin{array}{ll}
\tilde{U}^{c}\left(\hat{\eta}_{a I}, r\right)=\hat{a}_{a I}^{U} r^{2}+\hat{b}_{a I}^{U} r+\hat{c}_{a I}^{U} ; & \tilde{W}^{c}\left(\hat{\eta}_{a I}, r\right)=\hat{a}_{a I}^{W} r^{2}+\hat{b}_{a I}^{W} r+\hat{c}_{a I}^{W}, \\
\hat{a}_{a I}^{U}=a_{a}^{U} ; \quad \hat{b}_{a I}^{U}=b_{a}^{U}+2 a_{a}^{U} \hat{\eta}_{a I} ; \quad \hat{c}_{a I}^{U}=a_{a}^{U} \hat{\eta}_{a I}^{2}+b_{a}^{U} \hat{\eta}_{a I}+c_{a}^{U}, \\
\hat{a}_{a I}^{W}=a_{a}^{W} ; \quad \hat{b}_{a I}^{W}=b_{a}^{W}+2 a_{a}^{W} \hat{\eta}_{a I} ; \quad \hat{c}_{a I}^{W}=a_{a}^{W} \hat{\eta}_{a I}^{2}+b_{a}^{W} \hat{\eta}_{a I}+c_{a}^{W} .
\end{array}
$$

The resulting discretized integrand in (32) is given by

$$
\begin{aligned}
& \tilde{W}_{, r}^{c}\left(\hat{\eta}_{a I}, r\right) \tilde{U}_{\beta, r}^{\zeta}\left(\hat{\eta}_{a I}, r\right)-k_{\beta}^{2} \tilde{W}^{c}\left(\hat{\eta}_{a I}, r\right) \tilde{U}_{\beta}^{\zeta}\left(\hat{\eta}_{a I}, r\right) \\
& =i k_{\beta}\left(2 \hat{a}_{a}^{W} r+\hat{b}_{a}^{W}\right)\left(\hat{\tilde{A}}_{\beta} e^{i k_{\beta} r}-\hat{\tilde{B}}_{\beta} e^{-i k_{\beta} r}\right)-k_{\beta}^{2}\left(\hat{a}_{a}^{W} r^{2}+\hat{b}_{a}^{W} r+\hat{c}_{a}^{W}\right)\left(\hat{\tilde{A}}_{\beta} e^{i k_{\beta} r}+\hat{\tilde{B}}_{\beta} e^{-i k_{\beta} r}\right),
\end{aligned}
$$

where the coefficients $\hat{\tilde{A}}_{\beta}, \hat{\tilde{B}}_{\beta}$ of $\tilde{U}_{\beta}^{\zeta}$ are computed at a parent element domain for every integration point $\hat{\eta}_{e I}$ according to (18) from the following system

$$
\left[\begin{array}{cccc}
e^{-i k_{1} \zeta} & e^{i k_{1} \zeta} & 0 & 0 \\
0 & 0 & e^{i k_{2} \zeta} & e^{-i k_{2} \zeta} \\
e^{i k_{1} \zeta\left(\varphi_{1}-\varphi_{2}\right)} & e^{-i k_{1} \zeta\left(\varphi_{1}-\varphi_{2}\right)} & -e^{i k_{2} \zeta\left(\varphi_{1}-\varphi_{2}\right)} & -e^{-i k_{2} \zeta\left(\varphi_{1}-\varphi_{2}\right)} \\
z_{1} e^{i k_{1} \zeta\left(\varphi_{1}-\varphi_{2}\right)} & -z_{1} e^{-i k_{1} \zeta\left(\varphi_{1}-\varphi_{2}\right)} & -z_{2} e^{i k_{2} \zeta\left(\varphi_{1}-\varphi_{2}\right)} & z_{2} e^{-i k_{2} \zeta\left(\varphi_{1}-\varphi_{2}\right)}
\end{array}\right]\left[\begin{array}{c}
\hat{\tilde{A}}_{1} \\
\hat{\tilde{B}}_{1} \\
\hat{\tilde{A}}_{2} \\
\hat{\tilde{B}}_{2}
\end{array}\right]=\left[\begin{array}{c}
\tilde{U}^{c}\left(\hat{\eta}_{e I},-\zeta\right) \\
\tilde{U}^{c}\left(\hat{\eta}_{e I}, \zeta\right) \\
0 \\
0
\end{array}\right]
$$

Consequently, the dispersion relation (32) takes the form

$$
F(k, \omega)=\sum_{a=1}^{n^{e l}} \sum_{I=1}^{2} \sum_{\beta=1}^{2} v_{a l} \varphi_{\beta} z_{\beta}\left\langle\begin{array}{l}
i\left(2 \hat{a}_{a}^{W} r+\hat{b}_{e}^{W}\right)\left(\hat{\tilde{A}}_{\beta} e^{i k_{\beta} r}-\hat{\tilde{B}}_{\beta} e^{-i k_{\beta} r}\right) \\
-k_{\beta}\left(\hat{a}_{a}^{W} r^{2}+\hat{b}_{a}^{W} r+\hat{c}_{a}^{W}\right)\left(\hat{\tilde{A}}_{\beta} e^{i k_{\beta} r}+\hat{\tilde{B}}_{\beta} e^{-i k_{\beta} r}\right)
\end{array}\right\rangle_{\Theta_{a l}^{\prime \beta}}=0 .
$$

Here the frequency $\omega$ enters the dispersion relation (52) through $k_{\beta}=\omega \sqrt{\rho_{\beta} / E_{\beta}}$, $z_{\beta}=E_{\beta} k_{\beta}$ and equation (51). The wave number $k=2 \pi / \lambda$ appears in (52) through $d_{a \delta}^{U^{c}}, d_{a \delta}^{W^{c}}$ defined in (48), and is used in equations (47) and (51).

\section{A.3. Stress continuity and pseudo-periodicity for $\zeta=0.5$ or $\zeta=1$}

As mentioned in Appendix A.1, for two specific cases of the size ratio equal to $\zeta=0.5,1$ the two-point nonlocal quadrature (6)a is exact. In this Appendix, we further show that for these two cases the governing equation (30) reduces to stress continuity as well to the stress pseudoperiodicity at the unit cell boundaries, which make the dispersion relations of these cases identical to the reference solution. 
Recall that for $\zeta=0.5,1$ the boundaries of the computational unit cells coincide with the coarse-scale finite element nodes (see Remark 1 and Fig. 1), and consequently, we have $\tilde{U}^{c}\left(\hat{x}_{a I}, \pm l / 2\right)=U^{c}\left(\hat{x}_{a l}, \pm l / 2\right)$. Thus the overall displacement $U_{\beta}^{\zeta}$ is exact, due to (17) and (18).

Inserting the fine-scale problem (15) into the left-hand side of (30) and applying the chain rule yields

$$
\begin{aligned}
& \sum_{a=1}^{n^{a l}} \sum_{I=1}^{2} \sum_{\beta=1}^{2} \varphi_{\beta} E_{\beta}\left\langle W_{, \chi}^{c} U_{\beta, \chi}^{\zeta}+W^{c} U_{\beta, \chi \chi}^{\zeta}\right\rangle_{\Theta_{a l}^{\beta}}=\sum_{a=1}^{n^{a l}} \sum_{I=1}^{2} \sum_{\beta=1}^{2} \varphi_{\beta}\left\langle\left(W^{c} E_{\beta} U_{\beta, \chi}^{\zeta}\right)_{, \chi}\right\rangle_{\Theta_{a l}^{\beta}} \\
& =\frac{1}{l} \sum_{a=1}^{n^{l}} \sum_{I=1}^{2}\left[\left.\left(W^{c} E_{1} U_{1, \chi}^{\zeta}\right)\right|_{\chi_{\text {inf }}}-\left.\left(W^{c} E_{1} U_{1, \chi}^{\zeta}\right)\right|_{-\frac{l}{2}}+\left.\left(W^{c} E_{2} U_{2, \chi}^{\zeta}\right)\right|_{\frac{l}{2}}-\left.\left(W^{c} E_{2} U_{2, \chi}^{\zeta}\right)\right|_{\chi_{\text {inf }}}\right]=0 .
\end{aligned}
$$

Due to the continuity of stress $E_{\beta} U_{\beta, \chi}^{\zeta}$ and the coarse-scale test function $W^{c}$ at phase interfaces $\chi_{\text {inf }}$ (22),(28), equation (53) reduces to

$$
\sum_{a=1}^{n^{e l}} \sum_{I=1}^{2}\left[\left.\left(W^{c} E_{2} U_{2, \chi}^{\zeta}\right)\right|_{\frac{l}{2}}-\left.\left(W^{c} E_{1} U_{1, \chi}^{\zeta}\right)\right|_{-\frac{l}{2}}\right]=0,
$$

or

$$
\sum_{a=1}^{n^{a l}}\left[\left.\left(W^{c} E_{2} U_{2, \chi}^{\zeta}\right)\right|_{\hat{x}_{a 1}+\frac{l}{2}}-\left.\left(W^{c} E_{1} U_{1, \chi}^{\zeta}\right)\right|_{\hat{x}_{a 1}-\frac{l}{2}}+\left.\left(W^{c} E_{2} U_{2, \chi}^{\zeta}\right)\right|_{\hat{x}_{a 2}+\frac{l}{2}}-\left.\left(W^{c} E_{1} U_{1, \chi}^{\zeta}\right)\right|_{\hat{x}_{a 2}-\frac{l}{2}}\right]=0 .
$$

Since the boundaries of the computational unit cells coincide with element nodes, equation (54) can be rewritten as

$$
\sum_{a=1}^{n^{a l}}\left[\left.\left(W^{c} E_{2} U_{2, \chi}^{\zeta}\right)\right|_{d_{a 2}}-\left.\left(W^{c} E_{1} U_{1, \chi}^{\zeta}\right)\right|_{d_{a 1}}+\left.\left(W^{c} E_{2} U_{2, \chi}^{\zeta}\right)\right|_{d_{a 3}}-\left.\left(W^{c} E_{1} U_{1, \chi}^{\zeta}\right)\right|_{d_{a 2}}\right]=0 .
$$

where $d_{a \delta}$ is the nodal value at the $\delta$-th node of the $a$-th element. The terms corresponding to the first and last nodes vanish due to $\left.W^{c}\right|_{\partial \Omega^{c}}=0$. Furthermore, due to the arbitrariness of the test function and its continuity, equation (55) reduces to the stress continuity at the computational unit cell boundaries

$$
\begin{aligned}
& \left.\left(E_{1} U_{1, \chi}^{\zeta}\right)\right|_{d_{a 2}}=\left.\left(E_{2} U_{2, \chi}^{\zeta}\right)\right|_{d_{a 2}} ; \quad a=1, n^{e l}, \\
& \left.\left(E_{1} U_{1, \chi}^{\zeta}\right)\right|_{d_{(a+1) 1}}=\left.\left(E_{2} U_{2, \chi}^{\zeta}\right)\right|_{d_{a 3}} ; \quad a=1,\left(n^{e l}-1\right) .
\end{aligned}
$$

The stress continuity (56), along with displacement continuity (16) and the displacement pseudo-periodicity (26) at the unit cell boundaries, yield the stress pseudo-periodicity (21), which makes the dispersive $\mathrm{C}^{2}$ solution identical to the reference solution. Indeed, since the perturbation in (16) vanish at the unit cell boundaries, the total displacement is continuous, and thus

$$
U_{2}^{\zeta}\left(\hat{x}_{a I}, \frac{l}{2}\right)=U_{1}^{\zeta}\left(\hat{x}_{a I}, \frac{l}{2}\right)
$$

Inserting (57) into (26) yields 


$$
\begin{aligned}
& U_{1}^{\zeta}\left(\hat{x}_{a I}, \frac{l}{2}\right)=U_{1}^{\zeta}\left(\hat{x}_{a I},-\frac{l}{2}\right) e^{i k l}, \\
& U_{1, \chi}^{\zeta}\left(\hat{x}_{a I}, \frac{l}{2}\right)=U_{1, \chi}^{\zeta}\left(\hat{x}_{a I},-\frac{l}{2}\right) e^{i k l}, \\
& E_{1} U_{1, \chi}^{\zeta}\left(\hat{x}_{a I}, \frac{l}{2}\right)=E_{1} U_{1, \chi}^{\zeta}\left(\hat{x}_{a I},-\frac{l}{2}\right) e^{i k l} .
\end{aligned}
$$

Further enforcing the continuity of stress (56) to equation (58)c gives

$$
E_{2} U_{2, \chi}^{\zeta}\left(\hat{x}_{a I}, \frac{l}{2}\right)=E_{1} U_{1, \chi}^{\zeta}\left(\hat{x}_{a I},-\frac{l}{2}\right) e^{i k l}
$$

The above coincides with the pseudo-periodicity condition of stress (21).

Similarly, it can be shown that for four-node cubic elements with a three-point nonlocal quadrature (6)b and $\zeta=1 / 3$, stresses are continuous between the unit cell (that is, the pseudoperiodicity condition holds), which yields exact dispersion curves.

\section{References}

[1] B. Banerjee, An introduction to metamaterials and waves in composites, Taylor and Francis, 2011.

[2] J.D. Joannopoulos, R.D. Meade, J.N. Winn, Photonic crystals, Princeton Univ. Press, Princeton, 1995.

[3] C.T. Sun, J.D. Achenbach, G. Herrmann. Continuum theory for a laminated media. J. Appl. Mech. 35 (1968) 467-475.

[4] J.D. Achenbach, G. Herrmann. Dispersion of free harmonic waves in fiber-reinforced composites. AIAA J. 6 (1968) 1832-1836.

[5] A. Bedford, D.S. Drumheller, H.J. Sutherland. On modeling the dynamics of composite materials, in: Mechanics Today, Nemat-Nasser S (ed.), Vol. 3. Pergamon Press: New York, 1976

[6] V.I. Erofeev. Wave Processes in Solids with Microstructure. World Scientific: Singapore, 2003

[7] F. Santosa, W.W. Symes, A dispersive effective medium for wave propagation in periodic composites, SIAM J. Appl. Math. 51 (1991) 984-1005.

[8] C. Boutin, J.L. Auriault, Rayleigh scattering in elastic composite materials, Int. J. Eng. Sci. 31(1993) 1669-1689.

[9] J. Fish, W. Chen, Higher-order homogenization of initial/boundary-value problem, J. Eng. Mech. 127 (2001) 1223-1230.

[10] J. Fish, W. Chen, G. Nagai, Non-local dispersive model for wave propagation in heterogeneous media: multi-dimensional case, Int. J. Numer. Meth. Engng. 54 (2002) 347-363.

[11] N.S. Bakhvalov, M.E. Eglit, Equations of higher order accuracy describing the vibrations of thick plates, J. Appl. Math., Mech. 69 (2005) 593-610.

[12] I.V. Andrianov, V.I. Bolshakov, V.V. Danishevs'kyy, D. Weichert, Higher order asymptotic homogenization and wave propagation in periodic composite materials, Proc. R. Soc. A: Math. Phys. Eng. Sci. 464 (2008) 1181-1201.

[13] T. Hui, C. Oskay, A high order homogenization model for transient dynamics of heterogeneous media including micro-inertia effects, Comput. Methods Appl. Mech. Engrg. 273 (2014) 181-203.

[14] J.L. Auriault, C. Boutin, Long wavelength inner resonance cut-off frequencies in elastic composite materials, Int. J. Solids Struct. 49 (2012) 3269-3281.

[15] W. Chen, J. Fish. A dispersive model for wave propagation in periodic heterogeneous media based on homogenization with multiple spatial and temporal scales. J. Appl. Mech. 68 (2001) 153-161.

[16] J. Fish, W. Chen, Uniformly valid multiple spatial-temporal scale modeling for wave propagation in heterogeneous media, Mech. Compos. Mater. Struct. 8 (2001) 81-99. 
[17] E. Nolde, R. Craster, J. Kaplunov, High frequency homogenization for structural mechanics, J. Mech. Phys. Solids 59 (2011) 651-671.

[18] R.V. Craster, J. Kaplunov, A.V. Pichugin, High-frequency homogenization for periodic media, Proc. R.Soc. A: Math. Phys. Eng. Sci. 466 (2010) 2341-2362.

[19] S.K. Kanaun, V.M. Levin, Self-consistent methods in the problem of axial elastic shear wave propagation through fiber composites, Arch. Appl. Mech. 73 (2003) 105-130.

[20] S.K. Kanaun, V.M. Levin, F.J. Sabina, Propagation of elastic waves in composites with random set of spherical inclusions (effective medium approach), Wave Motion 40 (2004) 69-88.

[21] J. Willis, Effective constitutive relations for waves in composites and metamaterials, Proc. R. Soc. A: Math. Phys. Eng. Sci. 467 (2011) 1865-1879.

[22] S. Nemat-Nasser, A. Srivastava, Overall dynamic constitutive relations of layered elastic composites, J. mech. Phys., Solids 59 (2011) 1953-1965.

[23] A. Molinari, S. Mercier, Micromechanical modeling of porous materials under dynamic loading, J. Mech. Phys. Solids 49 (2001) 1497-1516.

[24] J. Fish, V. Filonova, S. Kuznetsov, Micro-inertia effects in nonlinear heterogeneous media, Int. J. Numer. Meth. Engng., 91 (2012) 1406-1462.

[25] Z.-P. Wang, Q. Jiang, A yield criterion for porous ductile media at high strain rate, J. Appl. Mech. 64 (1997) 503-509.

[26] K.Pham, V.G. Kouznetsova, M.G.D. Geers, Transient computational homogenization for heterogeneous materials under dynamic excitation, J. Mech. Phys. Solids 61 (2013) 2125-2146.

[27] W. Chen, J. Fish, A mathematical homogenization perspective of Virial stress, Int. J. Numer. Meth. Engng. 67 (2006) 189-207.

[28] G. Floquet, Sur les équations différentielles linéaires à coefficients périodiques. Ann. École Norm. Sup. 12 (1883) 47-88.

[29] L. Brillouin, Wave propagation in periodic structures: electric filters and crystal lattices, 1 st ed. New York, NY: Dover.

[30] R.L. Kronig, W.G. Penney, Quantum mechanics in crystal lattices, Proc. R. Soc. Lond. A: 130 (1931) 499-531.

[31] V.V. Vladimirskii, Propagation of radio waves along a chain of symmetric endovibrators, Doklady AN SSSR 52 (1946) 219-221. [In Russian]

[32] S.M. Rytov, Acoustical properties of a thinly laminated medium, Sov. Phys., Acoust. 2 (1956) 68-80.

[33] M.A. Silva, Study of pass and stop bands of some periodic composites, Acustica 75 (1991) 62-68.

[34] M. Ruzzene, A. Baz, Control of wave propagation in periodic composite rods using shape memory inserts J. Vib. Acoust. 122 (2000) 151-159.

[35] J. Fish, V. Filonova, D. Fafalis, Computational continua revisited, Int. J. Numer. Meth. Engng. (2014), DOI: 10.1002/nme.4793.

[36] J. Fish, Practical Multiscaling, Wiley, 2013.

[37] J. Fish, S. Kuznetsov, Computational continua, Int. J. Numer. Meth. Engng., 84 (2010) 774-802.

[38] R.J. Leveque, D.H. Yong, Solitary waves in layered nonlinear media, SIAM J. Appl. Math. 63 (2003) 1539-1560.

[39] F.V. Souza, D.H. Allen, Multiscale modeling of impact on heterogeneous viscoelastic solids containing evolving microcracks, Internat. J. Numer. Methods Engrg. 82 (2010) 464-504.

[40] I.V. Andrianov, V.V. Danishevs'kyy, O.I. Ryzhkov, D. Weichert, Dynamic homogenization and wave propagation in nonlinear 1d composite material, Wave Motion 50 (2012) 271-281.

[41] A. Karamnejad, L.J. Sluys, A dispersive multi-scale crack model for quasi-brittle heterogeneous materials under impact loading, Comput. Methods Appl. Mech. Engrg. 278 (2014) 423-444.

[42] T. J.R. Hughes, G. R. Feijóo, L. Mazzei, J.-B. Quincy, The variational multiscale method - a paradigm for computational mechanics, Comput.Methods Appl. Mech.Engrg. 166 (1998) 3-24. 
[43] D. Fafalis, J. Fish, "Wave propagation with Dispersive Computational Continua in elastic heterogeneous media", work in progress. 

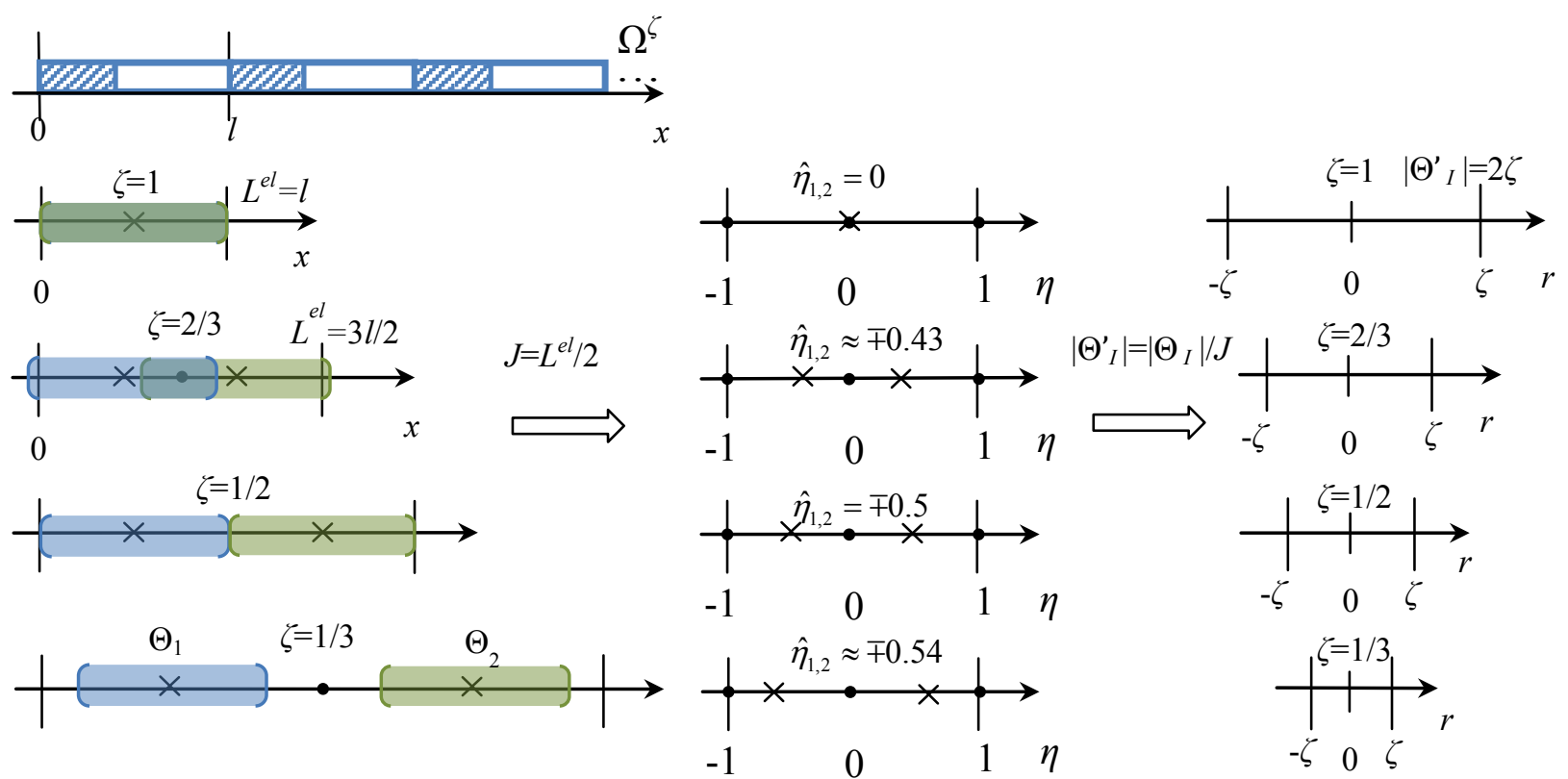

(a)

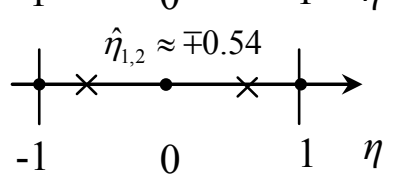

(b)

(c)

Fig.1 A three-node quadratic coarse-scale element with two integration points for various microstructures represented by the unit cell size ratio $\zeta=l / L^{e l}=1,2 / 3,1 / 2,1 / 3$ : (a) two "virtual" computational unit cells $\Theta_{1,2}$ (shown by blue and green colors) centered at the nonlocal quadrature points in the coarse-scale element domain; (b) nonlocal quadrature points in the parent element domain; and (c) the physical unit cell mapped onto the parent element domain in the local coordinate $r$. 


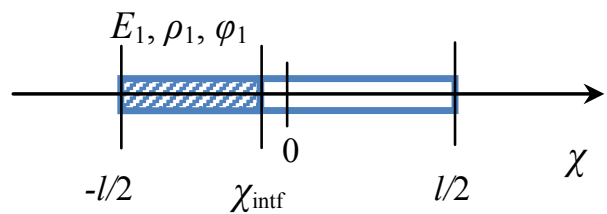

(a)

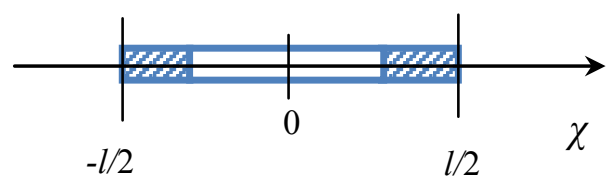

(b)

Fig. 2. Layered unit cell: non-symmetric (a) and symmetric (b) configurations. 


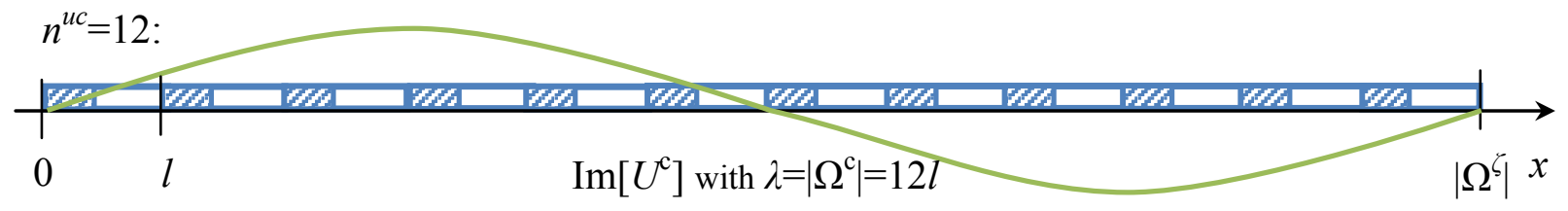

(a)
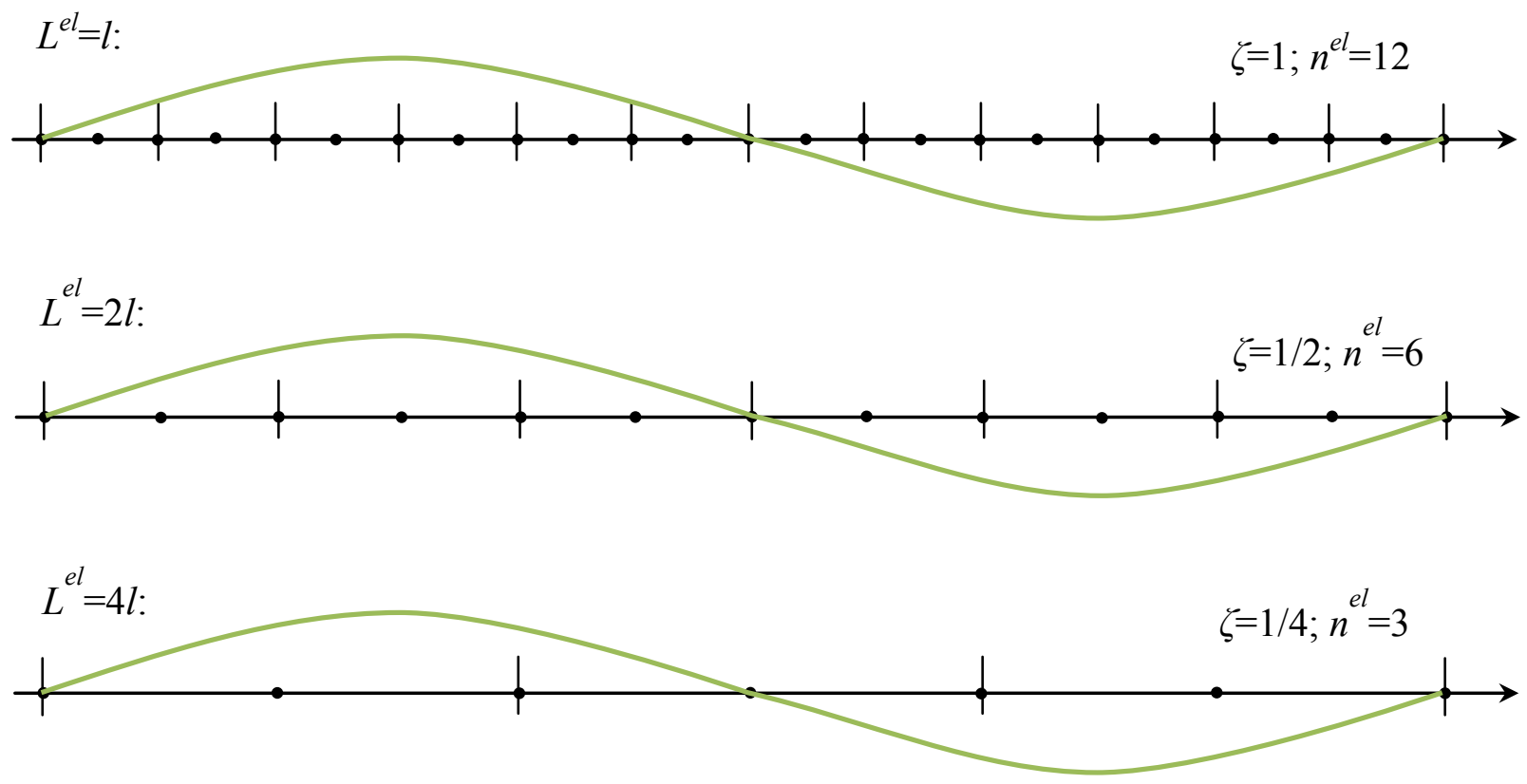

(b)

Fig. 3 (a) A composite domain consisting of a fixed number of unit cells $\left(n^{u c}=12\right.$ in the present example). The wave resolution $\operatorname{Im}\left[U^{c}\right]$ is shown for fixed $\lambda=\left|\Omega^{c}\right|$. (b) A coarse-scale domain discretization with $n^{e l}=12$ (top), $n^{e l}=6$ (middle) and $n^{e l}=3$ (bottom) elements with corresponding values of $\zeta=n^{e l} / n^{u c}=1,1 / 2,1 / 4$. 


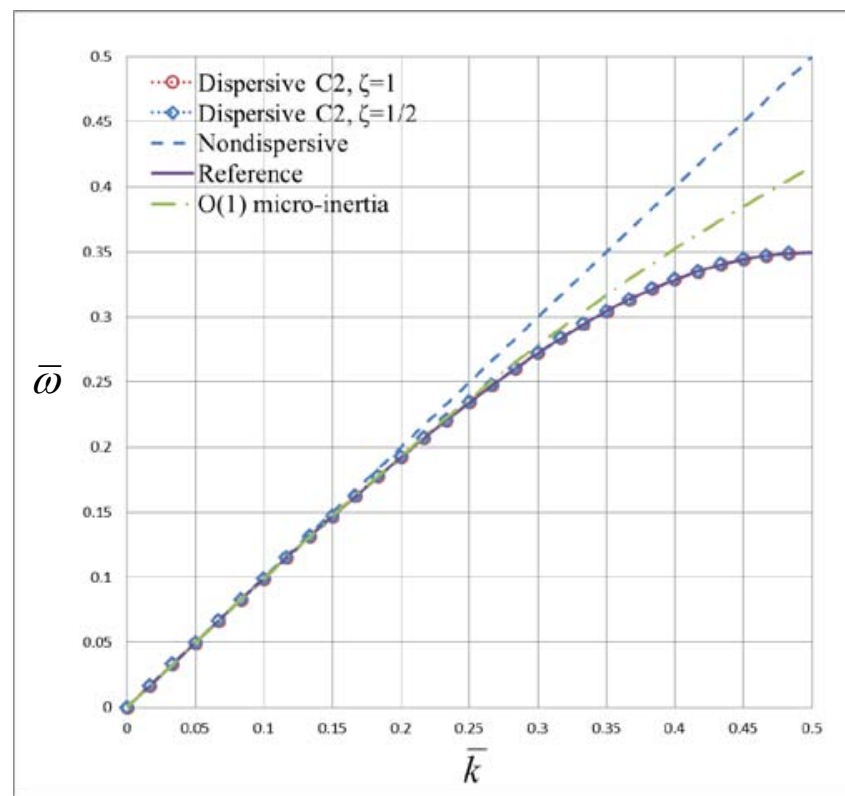

(a)

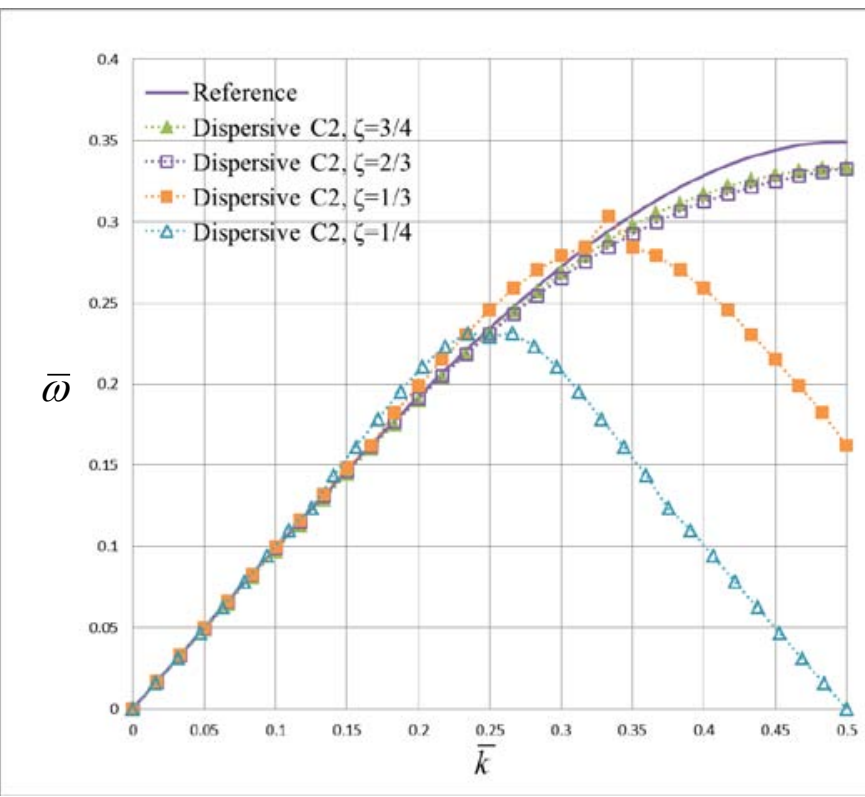

(b)

Fig. 4. Dispersion diagrams with $C^{2}$ results obtained by the analytical Floquet-Bloch approach. (a) Comparison of the nondispersive solution, dispersive $\mathrm{C}^{2}$, the $\mathrm{O}(1)$ micro-inertia solution, and the reference Floquet-Bloch dispersive curves for $\zeta=1 / 2,1$ and $n^{u c}=30$; (b) Comparison of the dispersive $C^{2}$ and the Floquet-Bloch dispersive curves for $\zeta=1 / 3,2 / 3$ and $n^{u c}=30, \zeta=1 / 4,3 / 4$ and $n^{u c}=32$. 


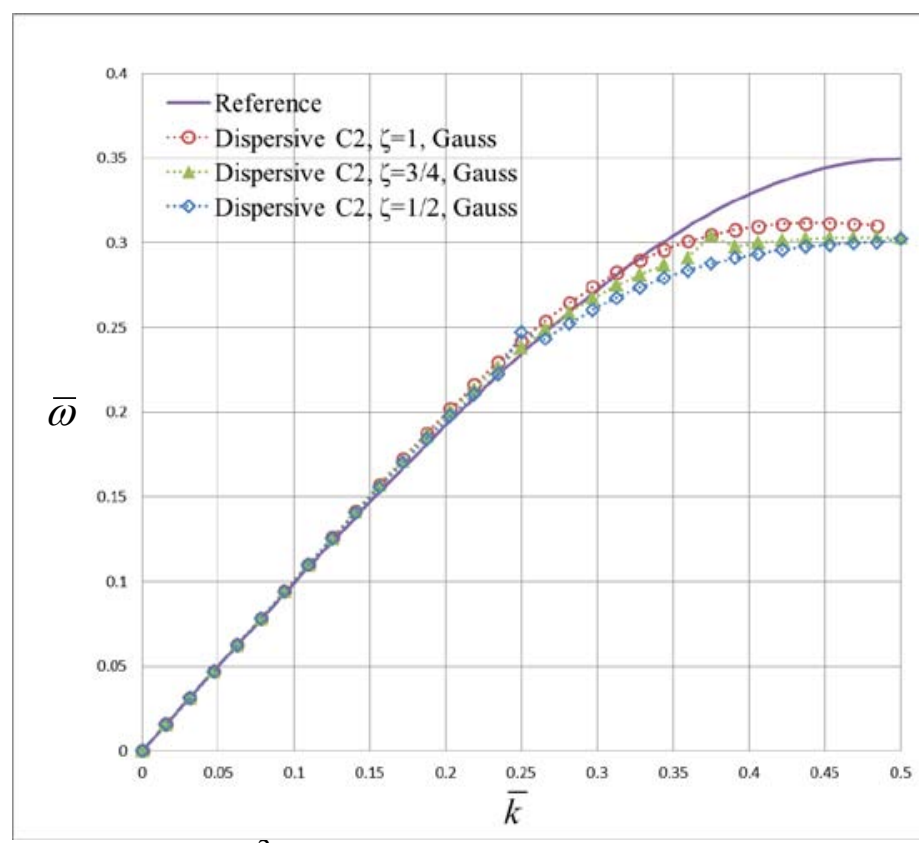

Fig. 5. Dispersion diagram with $C^{2}$; results obtained by the analytical Floquet-Bloch approach. Comparison of the dispersive $\mathrm{C}^{2}$ formulation obtained with the classical Gauss quadrature points and the Floquet-Bloch dispersive curves for $\zeta=1 / 2,3 / 4,1, n^{u c}=32$. 


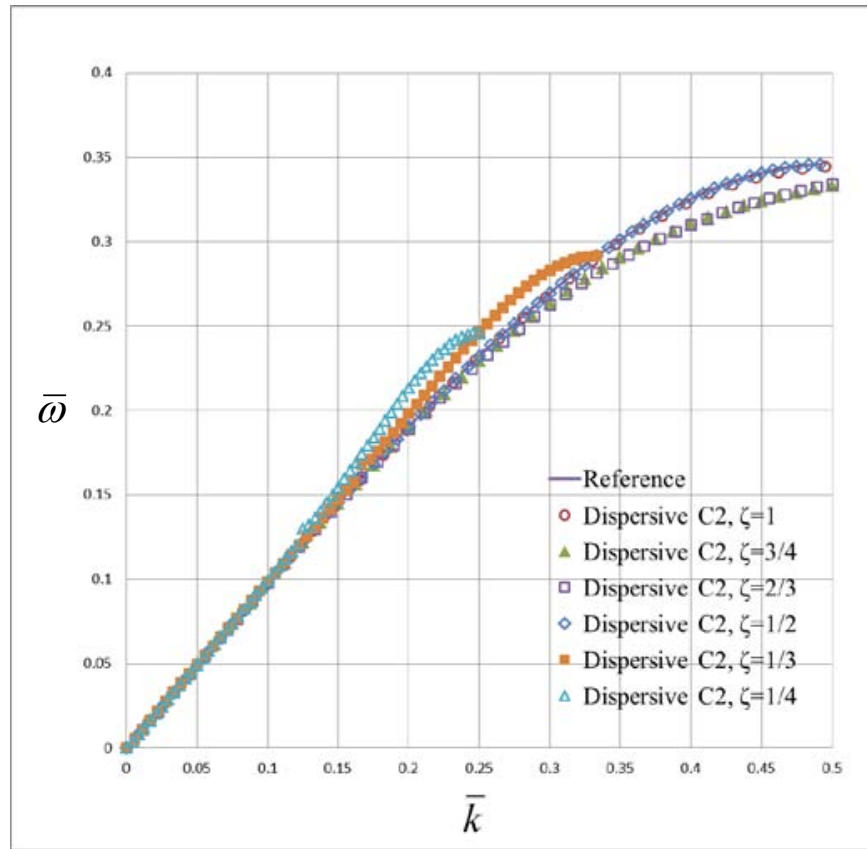

(a)

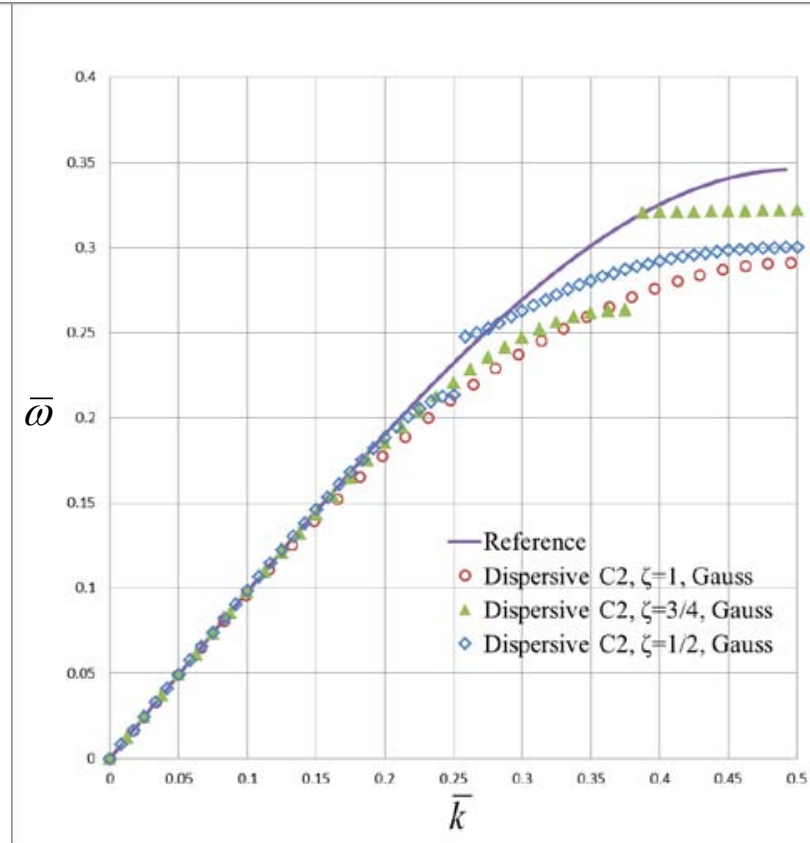

(b)

Fig. 6. Dispersion curves obtained from the modal analysis of the dispersive $C^{2}$ formulation (markers) and the reference Floquet-Bloch solution (solid line): (a) the dispersive $\mathrm{C}^{2}$ formulation for different values of $\zeta=1 / 4,1 / 3,1 / 2,2 / 3,3 / 4,1$; (b) the dispersive $C^{2}$ formulation employing classical Gauss quadrature points rather than the nonlocal quadrature points for $\zeta=1 / 2,3 / 4,1$. 


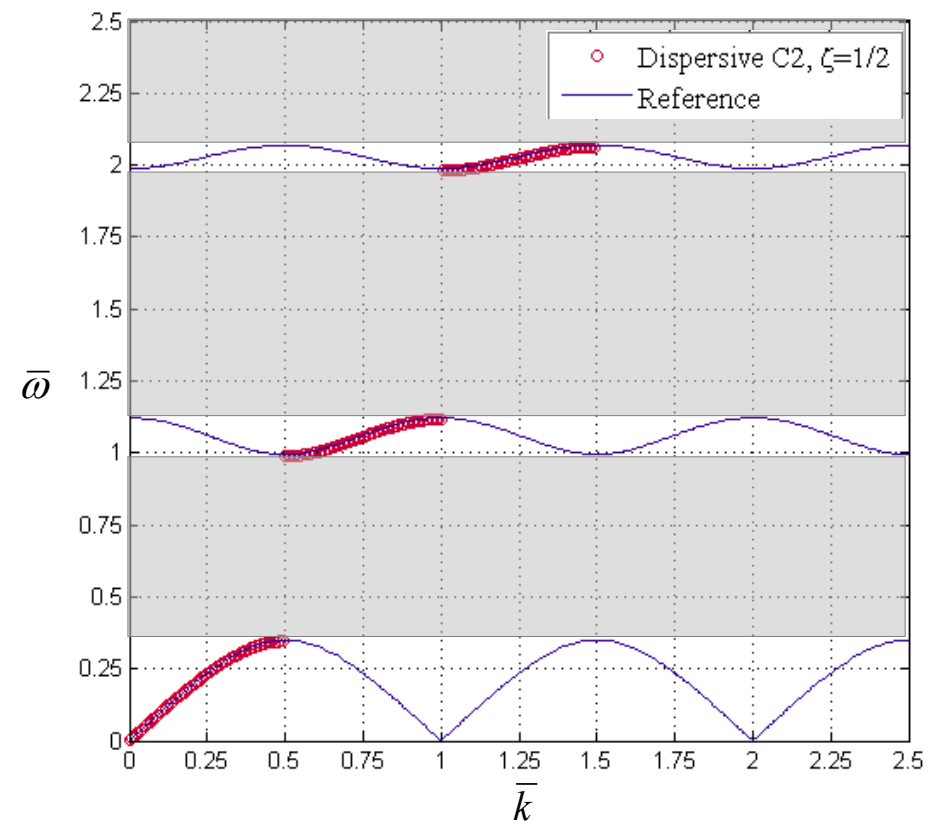

Fig. 7. Dispersion diagrams for the dispersive $C^{2}$ solution, obtained from the modal analysis, and the Floquet-Bloch solutions for a wide range of frequencies, $\zeta=1 / 2$. The grey areas indicate the stop frequency bands. 

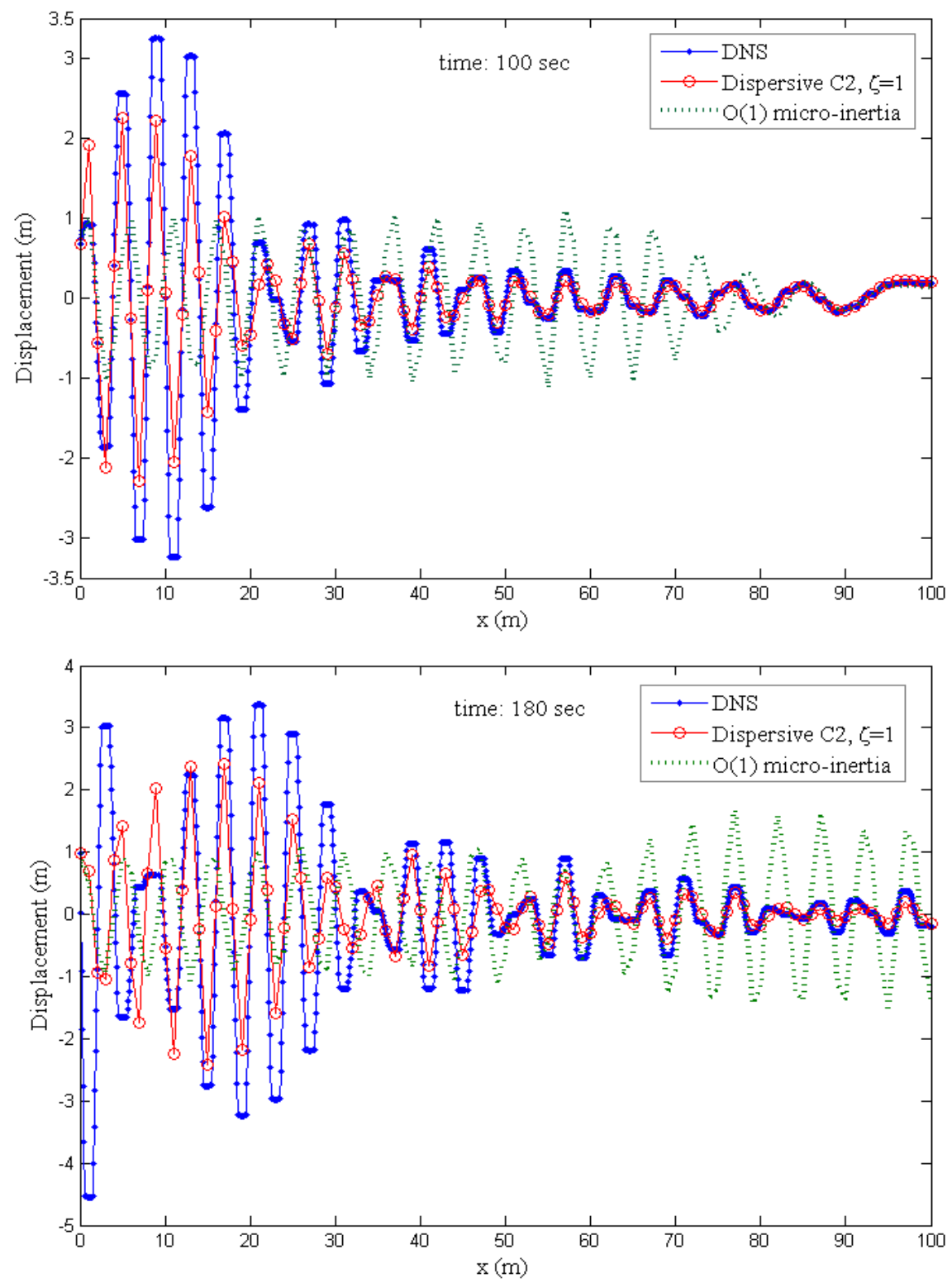

Fig. 8. The wave propagation in a heterogeneous rod. Comparison of the dispersive $C^{2}$, the $\mathrm{O}(1)$ micro-inertia and direct numerical simulation (DNS) for $\zeta=1$. 


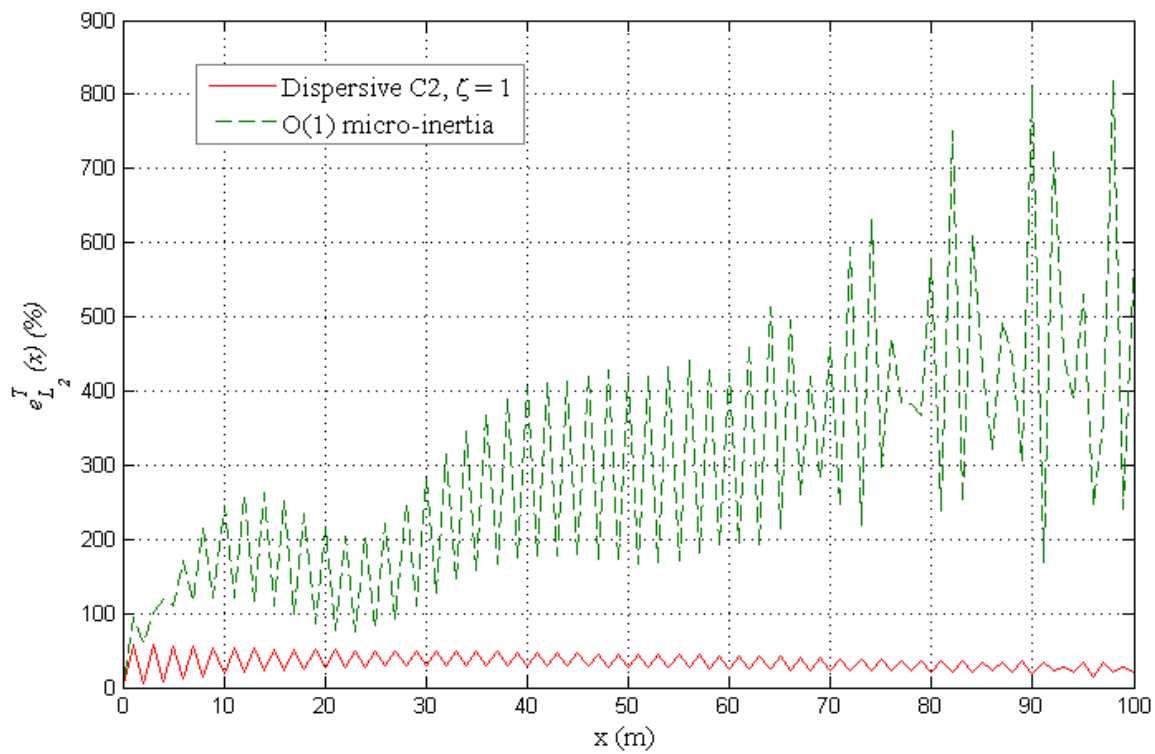

Fig. 9 The relative error $\bar{e}_{L_{2}}^{T}(x)$ computed over the time interval $t \in T=[0,200]$ shown over coarsescale domain. 